\title{
Uncertainties on the theoretical predictions for classical Cepheid pulsational quantities ${ }^{\star}$
}

\author{
G. Valle ${ }^{1}$, M. Marconi ${ }^{3}$, S. Degl'Innocenti ${ }^{1,2}$, and P. G. Prada Moroni ${ }^{1,2}$ \\ 1 Dipartimento di Fisica "Enrico Fermi”, Università di Pisa, largo Pontecorvo 3, Pisa 56127, Italy \\ 2 INFN, Sezione di Pisa, Largo B. Pontecorvo 3, Pisa 56127, Italy \\ 3 INAF, Osservatorio Astronomico di Capodimonte, via Moiariello 16, 80131 Napoli, Italy \\ e-mail: marcella@na.astro.it
}

Received 7 March 2009 / Accepted 27 August 2009

\begin{abstract}
Context. With their period-luminosity relation, "classical Cepheids" (CC) are the most common primary distance indicators within the Local Group, also providing an absolute calibration of important secondary distance indicators. However, the predicted position of these pulsators in the HR diagram, along the so called blue loop, that is the expected distribution of Cepheids within the instability strip is affected by several model inputs, reflecting upon the predicted $P L$ relation.

Aims. The aim of this work is to quantitatively evaluate the effects on the theoretical $P L$ relation of current uncertainties on the chemical abundances of Cepheids in the Large Magellanic Cloud (LMC) and on several physical assumptions adopted in the evolutionary models. We will separately analyse how the different factors influence the evolutionary and pulsational observables and the resulting $P L$ relation.

Methods. To achieve this goal we computed new sets of updated evolutionary and pulsational models.

Results. As a result, we find that present uncertainties on the most relevant $\mathrm{H}$ and $\mathrm{He}$ burning reaction rates do not influence in a relevant way the loop extension in temperature. On the contrary, current uncertainties on the LMC chemical composition significantly affect the loop extension and also reflect in the morphology of the instability strip; however their influence on the predicted pulsational parameters is negligible. We also discussed how overshooting and mass loss, sometimes suggested as possible solutions for the long-standing problem of the Cepheid mass discrepancy, influence the $M L$ relation and the pulsational parameters.

Conclusions. In summary, the present uncertainties on the physical inputs adopted in the evolutionary codes and in the LMC chemical composition are negligible for the prediction of the main pulsational properties. On the other hand, the inclusion of overshooting in the previous hydrogen burning phase and/or of mass loss is expected to significantly change the resulting theoretical pulsational scenario for Cepheids, as well as the calibration of their distance scale. These systematic effects are expected to influence the theoretical Cepheid calibration of the secondary distance indicators and in turn the resulting evaluation of the Hubble constant.
\end{abstract}

Key words. stars: variables: Cepheids - stars: evolution - stars: distances

\section{Introduction}

During the central helium burning phase, intermediate mass stars show an excursion toward higher effective temperature, in the Hertzprung-Russell (HR) diagram, subsequently coming back toward the asymptotic giant branch (blue loop). During this phase the stars cross the instability strip, becoming classical Cepheids. These variable stars, thanks to their characteristic period-luminosity $(P L)$ relation, are the most common primary distance indicators within the Local Group. Moreover, from space, they are observable also in external galaxies, up to distances of 20-30 Mpc (see e.g. Freedman et al. 2001). On this basis they provide the absolute calibration of important secondary distance indicators, such as the maximum luminosity of supernovae Ia, the Tully-Fisher relation, surface brightness fluctuations, and the planetary nebulae luminosity function, that are the basis for measurement of the Hubble constant (Freedman et al. 2001; Saha et al. 2001). Any systematic error affecting the Cepheid $P L$ relations affects the extragalactic distance scale and the estimate of the Hubble constant. Therefore, even though the Cepheid $P L$ relation is assumed to be universal and calibrated

$\star$ Tables $2,6,7,10,12,14,15,16$ are only available at http://www. aanda.org through the sample in the Large Magellanic Cloud (LMC, see e.g. Madore \& Freedman 1991; Udalski et al. 1999), the possibility that the Cepheid properties and $P L$ relation depend on the chemical composition of the parent galaxies has been actively debated during the last decade (see e.g. Sakai et al. 2004; Macri et al. 2006; Romaniello et al. 2005, 2008; Marconi et al. 2005; Bono et al. 2008). On the other hand the theoretical prediction for the existence of a $P L$ relation for classical Cepheids relies on the assumption that intermediate mass stars undergoing central helium burning are characterized by a mass-luminosity $(M L)$ relation, as predicted by stellar evolution models. On very general grounds the pulsation period is expected to be tightly correlated with the luminosity, the mass and the effective temperature of the star, for each given chemical composition. It is only thanks to the $M L$ relation that the period can be simply related to the luminosity and the effective temperature, allowing a theoretical prediction for the period-luminosity-color relation $(P L C$, see Feast 1984, 1991, 1995; Caldwell \& Coulson 1986; Laney \& Stobie 1986; Stothers 1988; Caldwell \& Laney 1991; Sasselov et al. 1997). However the theoretical $M L$ relation depends on the several physical processes included in stellar evolutionary codes as well as on the adopted chemical composition. 
By averaging the $P L C$ relation on the color extension of the pulsation instability strip one obtains the $P L$ relation. This implies that the $P L$ relation has a statistical nature and observationally holds only when a statistically significant sample of Cepheids (at the same distance) is available. Thus the predicted temperature extension of the blue loop for a given mass also reflects upon the expected distribution of Cepheids within the instability strip and in turn upon the theoretical $P L$ relation. This is a very critical point because the predicted extension of the blue loop in the HR diagram is affected by several model inputs (chemical composition, efficiency of external convection, nuclear burning cross sections, etc.) producing effects that are not yet completely understood (see e.g. Xu \& Li 2004a; Stothers \& Chin 1994; Brunish et al. 1990). In this context, a detailed comparison of evolutionary and pulsational models with the observations can provide insight into the physics of stellar evolution and pulsation.

The aim of this work is to quantitatively evaluate the effects on the predicted $P L$ relation of current uncertainties on the chemical abundances of Cepheids in the LMC and of several physical assumptions adopted in the evolutionary models. In particular, we will separately analyse how the different factors influence the evolutionary and pulsational observables (extension in effective temperature of the blue loop, Mass-Luminosity relation, morphology of the instability strip, period distribution) and the resulting $P L$ relation. For the chemical composition we choose that of the LMC with its associated uncertainty. All the relations based on the newly computed sets of evolutionary and pulsation models will be made available to the community. In a forthcoming paper (Valle et al. 2009, in preparation) we will focus on the comparison of these new predictions with the observations. The organization of the paper is the following: in Sect. 2 we present the theoretical scenario both from the evolutionary and the pulsational point of view; Sect. 3 contains a general discussion about the problems in calculating blue loop models; in Sect 4 the dependence of the predicted extension of the blue loop on the uncertainties affecting nuclear reaction rates is investigated; in Sect. 5 we focus on the effects of chemical abundance uncertainties on the most relevant evolutionary and pulsational features, whereas in Sect. 6.1 we take into account possible noncanonical effects in the evolutionary scenario. The conclusions, reported in Sect. 7, close the paper.

\section{Theoretical scenario}

In this section we present the new sets of evolutionary and pulsation models that have been used for the calculations.

\subsection{Evolutionary stellar models}

Evolutionary tracks were computed with an updated version of the FRANEC evolutionary code (see e.g. Chieffi \& Straniero 1989; Degl'Innocenti et al. 2008) including the OPAL 2006 equation of state $(\mathrm{EOS})^{1}$ (see also Rogers et al. 1996) and radiative opacity tables ${ }^{2}$ (see also Iglesias \& Rogers 1996) for temperatures higher than $12000 \mathrm{~K}$. Moreover our models include the conductive opacities by Shternin \& Yakovlev (2006) (see also Potekhin 1999) and the atmospheric opacities by Ferguson et al. $(2005)^{3}$. All the adopted opacity tables have been calculated by

\footnotetext{
1 http://www-phys.1lnl.gov/Research/OPAL/EOS_2005/

2 http://www-phys.1lnl.gov/Research/OPAL/opal.html

3 Available at http://webs.wichita.edu/physics/opacity
}

assuming the solar relative metal abundances by Asplund et al. (2005).

Since the ${ }^{12} \mathrm{C}(\alpha, \gamma){ }^{16} \mathrm{O}$ and ${ }^{14} \mathrm{~N}(p, \gamma){ }^{15} \mathrm{O}$ reaction rates affect the evolutionary properties of intermediate-mass stars and in particular the temperature blue loop extension (see e.g. Brunish et al. 1990), we adopted the updated rates by Hammer et al. (2005) for the ${ }^{12} \mathrm{C}+\alpha$ and by the LUNA collaboration (see Imbriani et al. 2005, and references therein) for the ${ }^{14} \mathrm{~N}+p$ cross sections. For the other nuclear reactions we adopted the same rates as in Ciacio et al. (1997). The code implements weak screening (Salpeter 1954), weak-intermediate, intermediate-strong screening (Graboske et al. 1973), and strong screening (Itoh et al. 1977, 1979).

For convective mixing, we adopt the Schwarzschild criterion to define regions in which convection elements are accelerated (see the description in Brocato et al. 2003) with an additional algorithm to take into account the induced semiconvection during the central He-burning phase (Castellani et al. 1971a,b). Rotationally induced effects (see e.g. Maeder \& Zahn 1998; Palacios et al. 2003) are not included in our models.

To model envelope convection we adopted, as usual, the mixing length formalism (Bohm-Vitense 1958). The mixing length parameter, $\alpha$, governing the efficiency of convection, has been calibrated to reproduce the observed stellar colors in the Large Magellanic Cloud cluster NGC 1866, obtaining $\alpha=1.9$ (Brocato et al. 2004). This result is also dependent on the atmospheric models adopted to transform evolutionary calculations from the theoretical $\left(\log L-\log T_{\mathrm{e}}\right)$ to the observational plane. In this paper we adopted the color-temperature transformations by Bessel et al. (1998).

The original helium mass fraction $(Y)$ cannot be measured spectroscopically and it is generally estimated by assuming a linear relation between $Y$ and $Z$ :

$Y=Y_{\mathrm{P}}+\frac{\Delta Y}{\Delta Z} \times Z$

where $Y_{\mathrm{P}}$ is the primordial helium abundance and $\Delta Y / \Delta Z$ is the estimated helium-to-metal enrichment ratio. For $Y_{\mathrm{P}}$ we adopted the value $Y_{\mathrm{P}}=0.248$ (Peimbert et al. 2007; Izotov et al. 2007) and $\Delta Y / \Delta Z=2$ (Jimenez et al. 2003).

The metallicity adopted for the models is consistent with the most recent estimates of $[\mathrm{Fe} / \mathrm{H}]$ for LMC Cepheids (see Luck et al. 1998; Romaniello et al. 2008) and also with several independent determinations of the LMC metallicity (see e.g. Korn et al. 2000; Andrievsky et al. 2001).

The total metallicity can be expressed as follows:

$Z=\left(1-Y_{\mathrm{P}}\right)\left(1+\frac{\Delta Y}{\Delta Z}+\frac{1}{(Z / X)_{\odot}} \times 10^{-[\mathrm{Fe} / \mathrm{H}]}\right)^{-1}$

where $(Z / X)_{\odot}$ is the solar metal to hydrogen abundance ratio.

In this work we adopt the updated Asplund et al. (2005) mixture, based on the analysis of spectroscopic data by means of tridimensional hydrodynamical atmospheric models, instead of that by Grevesse \& Noels (1993), hereinafter GN93, widely used in the past. This leads to a significant reduction of the the $Z / X$ ratio for the Sun, from $Z / X \approx 0.0245$ to $Z / X \approx 0.0165$. As a result the traditionally assumed metallicity for LMC decreases from $Z \approx 0.008$ to $Z \approx 0.005$. With this choice of metallicity, from Eq. (1), one obtains $Y \approx 0.26$, which we assume as our standard value.

Several investigations have dealt with the analysis of the effects of this revision of the solar abundance, revealing discrepancies between predicted and helioseismologically inferred quantities (see e.g. Bahcall et al. 2005; Basu \& Antia 2004). This 
situation is further complicated by the fact that current uncertainties on the physical inputs adopted in the solar models can affect the results of the quoted investigations. Moreover some authors questioned the accuracy of the oxygen abundance derived by Asplund et al. (2005) (see e.g. Caffau et al. 2008). For these reasons, even if we adopted the Asplund et al. (2005) solar mixture as our reference mixture, in the following we will take into account the uncertainty related to this assumption and its influence on the LMC metallicity.

\subsection{Nonlinear pulsation models}

Our pulsational models are based on a nonlinear nonlocal time-dependent convective hydrodynamical code (Bono \& Stellingwerf 1994; Bono et al. 1999a, and references therein). This code has been successfully applied to several classes of pulsating stars (see e.g. Marconi et al. 2003; Di Criscienzo 2004; Bono et al. 1999b; Marconi et al. 2004, 2005; Marconi \& Di Criscienzo 2007, and references therein). In this paper the physical ingredients of Cepheid pulsation models, and in particular the stellar opacity tables, have been updated in order to insure consistency between the pulsation and the evolutionary models. The new pulsation models have been constructed with the same chemical abundances of evolutionary models, covering the same range in stellar mass and assuming the luminosity predicted by the canonical Mass-Luminosity $(M L)$ relation by Bono et al. (2000). For $Z=0.005$, a second luminosity brighter by 0.25 dex has been included to account for the effect of mild overshooting and mass loss (see Sect. 6.1).

\section{Modeling the blue loop morphology}

The extension in temperature of the blue loop is an important evolutionary feature. Stellar models show that the excursion to the blue is produced by an envelope contraction (see e.g. Renzini et al. 1992): models with more extended loops undergo a more significant envelope contraction which heats the hydrogen shell, increasing its luminosity. The return of the track to the red is due to a variety of effects (see e.g. Sestito et al. 2002) : a) when the central helium abundance decreases below $Y_{\mathrm{c}} \sim 0.1$ the star reacts to the decreased burning rate by means of a core contraction increasing again the energy production and thus the amount of energy reaching the envelope, which expands and cools; b) the $\mathrm{H}$ shell reaches the discontinuity in the chemical profile left by the first dredge up before the core contraction (see e.g. the discussion in Lauterborn et al. 1971; Huang \& Weigert 1983; Sestito et al. 2002; Xu \& Li 2004a). However, as widely discussed in the literature (see e.g. Stothers \& Chin 1993; Alcock \& Paczynski 1978; Robertson 1971; Brunish et al. 1990; Sestito et al. 2002; Xu \& Li 2004b), the formation and the extension of loops appear to be caused by the interaction of several factors and is extremely sensitive to small changes either in the stellar chemical composition or in the physical inputs adopted in the calculations. Several authors have discussed the poor stability naturally existing in many stellar models which lie on the red supergiant branch during the central He burning phase, that is, around the beginning of the blue loop (see e.g. Noels \& Gabriel 1973; Stothers \& Chin 1991a).

The dependence of the loop morphology on changes in the chemical composition will be analysed in Sect. 5. In general, an increase of the original $\mathrm{He}$ abundance increases the mean molecular weight while it leads to a decrease of the mean opacity (see e.g. Vemury \& Stothers 1978), even if the dependence on He abundance is not linear with metallicity. On the other hand a metallicity variation changes the blue loop extension mainly due to the opacity variation (see e.g. Stothers \& Chin 1993) even if the behaviour can be more complicated and a quantitative explanation of the loop dependence on chemical composition is still lacking in the literature.

The evolutionary properties of intermediate and high mass stars in the central He burning phase strongly depend on the efficiency of the He burning nuclear reactions (see e.g. Iben 1972; Brunish et al. 1990; Bono et al. 2000). This point is discussed in the following subsections in the light of updated nuclear cross sections measurements.

Regarding the opacity dependence, any increase of the opacities deep in the stellar interiors could lead to fainter luminosities, cooler effective temperatures and longer lifetimes. Regarding the loop extension, in principle, a higher opacity leads to a reduced blue loop but the effect is in general very small (see e.g. Stothers \& Chin 1991b). In addition, updated opacity tables agree amongst each other within 5-10\% (see e.g. discussion in Badnell et al. 2005; Neuforge-Verheecke et al. 2001) thus very small opacity uncertainties are expected. Moreover, molecular opacities have almost negligible effects on the blue loop extension (see e.g. Stothers \& Chin 1993).

Several authors (see e.g. Chiosi et al. 1992; Stothers \& Chin 1992; Brocato \& Castellani 1993) noticed that the extension of the convective $\mathrm{H}$ burning core affects the following He burning phase. This involves the convective core overshooting efficiency, whose influence on the blue loop extension will be discussed in detail in Sec. 6.1 and the method adopted for treating the semiconvective layers that develop around the $\mathrm{H}$ burning core close to the central $\mathrm{H}$ exhaustion. If a significant amount of overshooting is included in the calculations, during the core He burning phase the luminosity is brighter, the lifetime is shorter and the blue loop is less extended and rises less steeply than in absence of overshooting (see e.g. Matraka et al. 1982; Chin \& Stothers 1991).

The possible occurrence of convective shells and the consequent modification of the internal chemical profile are strictly related to the adopted stability criterion, either Schwarzschild or Ledoux (see e.g. Salasnich et al. 1999; Stothers \& Chin 1994; Canuto 2000). However the stability criterion has a marginal effect on stellar models ranging from $\approx 3 M_{\odot}$ to $\approx 12 M_{\odot}$, as the ones taken into account in our work Stothers \& Chin (1993).

Moreover downward convecting overshooting from the outer convection zone which modifies the envelope chemical profile influences the formation of blue loops.

For the same reason the occurrence of blue loops is thus influenced by the mixing length value $\alpha$, which governs the convection efficiency in the external convective envelope. However when a blue loop develops (it is not true in some cases of small and moderate $\alpha$ values) the maximum effective temperature during the blue loop was found to be only a very weak function of $\alpha$ (see e.g. Chin \& Stothers 1991). Moreover, since the atmospheric model is fixed, $\alpha$ is generally calibrated through the comparison between theory and observation for the stellar color. For this reason, in this paper we will not include variations of this parameter, which is fixed to $\alpha=1.9$.

Another important phenomenon affecting the evolution of He burning intermediate mass stars is mass loss (see e.g. the review by Chiosi \& Maeder 1986 or the more recent work by Salasnich et al. 1999). As discussed by several authors (see e.g. Salasnich et al. 1999) mass loss mainly influences the evolution of masses higher than $\approx 15 M_{\odot}$ but its influence on the loop morphology, as discussed in Sect. 6.2, can be significant also for lower masses. However the physical mechanisms which 
affect mass loss and its dependence on metallicity are not still completely understood and thus the range of possible efficiencies for the phenomenon is very wide (see e.g. the discussion in Maeder \& Meynet 2000; Vázquez et al. 2005). In this paper we work in the canonical regime, also because we concentrate on intermediate mass stars but to take into account a possible mass loss contribution, in Sect. 6.2 we discuss some additional models computed with different mass loss efficiencies.

Due to the dependence of the loop extension on several physical parameters, if one compares recent sets of intermediate mass stars models available in the literature (which in general differ by the assumed chemical composition, the adopted physical inputs, the external convection efficiency etc.) one finds significant variations in the loop morphology. In the following sections we will discuss the main parameters which have already been demonstrated to potentially affect in a relevant way the blue loop morphology: He burning cross sections, chemical composition, overshooting and mass loss efficiency.

\section{Dependence of the loop extension on the uncertainties on the nuclear reaction cross sections}

Several authors have investigated the dependence of the loop extension on the $\mathrm{H}$ burning (through the $\mathrm{CNO}$ cycle) and the He burning reaction rates (see e.g. Brunish et al. 1990; Renzini et al. 1992; Sestito et al. 2002; Xu \& Li 2004a; Weiss et al. 2005), and have shown that the loop morphology is sensitive to variations of the adopted cross sections.

In this section we focus on the dependence of the loop formation and its temperature extension on variations of the relevant nuclear fusion cross sections within their estimated uncertainties. For stars in the central He burning phase, the most relevant nuclear reactions are the $3 \alpha$, the ${ }^{12} \mathrm{C}(\alpha, \gamma){ }^{16} \mathrm{O}$, that affects the He burning efficiency and the ${ }^{14} \mathrm{~N}(p, \gamma){ }^{15} \mathrm{O}$ that governs the shell $\mathrm{H}$ burning through the $\mathrm{CNO}$ cycle.

\section{1. ${ }^{14} \mathrm{~N}(p, \gamma)^{15} \mathrm{O}$ cross section}

In stars with internal temperatures higher than $\approx 2 \times 10^{7} \mathrm{~K}$, the hydrogen burning is dominated by the $\mathrm{CNO}$ cycle whose rate is determined by the slowest process, the ${ }^{14} \mathrm{~N}(p, \gamma){ }^{15} \mathrm{O}$ reaction. For this reaction the Gamow peak is at $\approx 30 \mathrm{keV}$; thus the capture cross section must be known down to this energy at which it has a very low value, preventing a direct laboratory measurement. Instead, the cross sections are measured at higher energies and then extrapolated, by means of the astrophysical $\mathrm{S}(\mathrm{E})$ factor, down to the Gamow peak region. The occurrence of resonance and resonance tails complicates the extrapolation and it results in very large uncertainties on the evaluated cross sections. Recently, the ${ }^{14} \mathrm{~N}(p, \gamma){ }^{15} \mathrm{O}$ cross section has been measured by the LUNA collaboration close to $70 \mathrm{keV}$ (see e.g. Imbriani et al. 2005; Bemmerer et al. 2006; Lemut et al. 2006), leading to an improved estimate of the $S(E)$ factor at astrophysical energies $(S(0)=1.61 \pm 0.08 \mathrm{keV}$, about half of the value of the NACRE compilation by Angulo et al. 1999).

To quantify the effect of a variation of the ${ }^{14} \mathrm{~N}+p$ cross section, we computed stellar models for several stellar masses, varying the ${ }^{14} \mathrm{~N}+p$ reaction rate of the estimated uncertainty with respect to the LUNA value (adopted in our standard models), models IX and X of Table 1. For all the investigated cases the effect on the loop morphology of the present uncertainty on the ${ }^{14} \mathrm{~N}(p, \gamma){ }^{15} \mathrm{O}$ rate is negligible, suggesting that this uncertainty is
Table 1. Computed sets of evolutionary tracks and pulsation models.

\begin{tabular}{llll}
\hline \hline Case & $Y$ & $Z$ & Mixture \\
\hline I $(s t d)$ & 0.26 & 0.005 & AGS05 \\
II $\left(Z_{\text {low }}\right)$ & 0.26 & 0.0035 & AGS05 \\
III $\left(Z_{\text {high }}\right)$ & 0.26 & 0.008 & AGS05 \\
IV $\left(Y_{\text {high }}\right)$ & 0.28 & 0.005 & AGS05 \\
V $\left(Y_{\text {high }}, Z_{\text {high }}\right)$ & 0.28 & 0.008 & AGS05 \\
VI $\left(Z_{\text {high }}, \mathrm{GS} 98\right)$ & 0.26 & 0.008 & GS98 \\
VII $\left({ }^{12} \mathrm{C}(\alpha, \gamma)^{16} \mathrm{O}\right)_{\text {high }}$ & 0.26 & 0.005 & AGS05 \\
VIII $\left({ }^{12} \mathrm{C}(\alpha, \gamma){ }^{16} \mathrm{O}\right)_{\text {low }}$ & 0.26 & 0.005 & AGS05 \\
IX $\left({ }^{14} \mathrm{~N}(p, \gamma)^{15} \mathrm{O}\right)_{\text {high }}$ & 0.26 & 0.005 & AGS05 \\
X $\left({ }^{14} \mathrm{~N}(p, \gamma)^{15} \mathrm{O}\right)_{\text {low }}$ & 0.26 & 0.005 & AGS05 \\
XI $\left(l_{\text {ov }}=0.1 H_{\mathrm{p}}\right)$ & 0.26 & 0.005 & AGS05 \\
XII $\left(l_{\text {ov }}=0.25 H_{\mathrm{p}}\right)$ & 0.28 & 0.005 & AGS05 \\
XIII $\left(l_{\text {ov }}=0.25 H_{\mathrm{p}}\right)$ & 0.26 & 0.005 & AGS05 \\
\hline
\end{tabular}

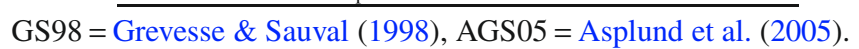

not expected to contribute significantly to the final error budget for the predicted Cepheid pulsation properties.

\section{2. $3 \alpha$ cross section}

Recently Fynbo et al. (2006) reported new measurements for the $3 \alpha$ rate, finding, in particular, a resonance at energies of $\approx 11 \mathrm{MeV}$; however, in the temperature range of interest, the differences with respect to the most widely adopted $3 \alpha$ rate are within the uncertainty evaluated by the NACRE (Angulo et al. 1999 ) compilation, reaching a maximum of $\approx 20 \%$ at temperatures of about $10^{8} \mathrm{~K}$ (see e.g. Weiss et al. 2005).

For this reason we decided to take the error quoted by NACRE (20\%), in the temperature range of interest, as a conservative estimate of the uncertainty on the $3 \alpha$ reaction rate. We computed specific models by varying the $3 \alpha$ rate within the estimated uncertainty for different stellar masses (in our "standard" case) finding no relevant variations in the loop extension. These results suggest that the present uncertainties on the $3 \alpha$ rate do not substantially influence the evolutionary and pulsational quantities for Cepheids stars.

\section{3. ${ }^{12} \mathrm{C}(\alpha, \gamma){ }^{16} \mathrm{O}$ cross section}

The measurement of the ${ }^{12} \mathrm{C}+\alpha$ cross section at the energies of interest in stars $(\approx 300 \mathrm{keV})$ is presently impossible due to the smallness of the predicted value $\left(\simeq 10^{-17} \mathrm{~b}\right)$. The extrapolation from higher energies is quite difficult, because the cross section in this energy region is a mixture of groundstate and cascade transitions, and the cross section for the ground state transitions is dominated by the tails of subthreshold resonances with the interference of other processes (see e.g. Buchmann et al. 2006). For these reasons the uncertainty quoted in the past for this rate was very large, up to a factor of two, and the effect of this error on the loop extension was very strong (see e.g. Brunish et al. 1990). Given the astrophysical importance of this reaction, in the past decade a significant experimental effort has been devoted to the improvement of the determination of its cross section. Recent results by Hammer et al. (2005) of direct ${ }^{12} \mathrm{C}(\alpha, \gamma){ }^{16} \mathrm{O}$ measurements in the temperature range $0.001 \leq T_{9} \leq 10, T_{9}$ being the temperature in $10^{9} \mathrm{~K}$, lead to a new determination of the extrapolated astrophysical reaction rate with a maximum total uncertainty of $\pm 25 \%$ (see Hammer et al. 2005).

In the present work we adopt for the ${ }^{12} \mathrm{C}(\alpha, \gamma){ }^{16} \mathrm{O}$ reaction rate the parametrization and the parameter values given by 


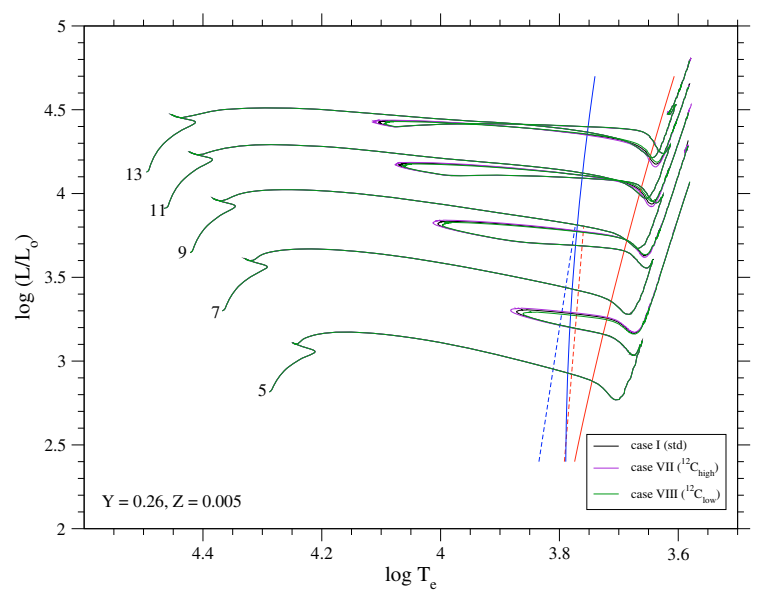

Fig. 1. Evolutionary tracks with standard chemical composition with three different values of the ${ }^{12} \mathrm{C}(\alpha, \gamma)^{16} \mathrm{O}$ rate (see text).

Hammer et al. (2005); at the typical He burning temperature $\left(10^{8} \div 2 \times 10^{8} \mathrm{~K}\right)$ this new rate is about $20 \%$ lower than the NACRE rate.

As discussed in Brunish et al. (1990), the blue loop extension depends on the ${ }^{12} \mathrm{C}+\alpha$ cross section: the higher the rate of this reaction, the higher the effective temperature excursion of the loop. Several authors (see e.g. Sestito et al. 2002; Xu \& Li 2004a) showed that the ${ }^{12} \mathrm{C}(\alpha, \gamma){ }^{16} \mathrm{O}$ cross section is related to the efficiency of the envelope contraction and thus to the loop extension.

We evaluated the influence on the loop extension of the present uncertainty on the ${ }^{12} \mathrm{C}(\alpha, \gamma){ }^{16} \mathrm{O}$ reaction rate for models of different masses. To this aim we calculated two additional sets of tracks with "standard" chemical composition $(Z=0.005$ $Y=0.26$ ) and the ${ }^{12} \mathrm{C}+\alpha$ rate by Hammer et al. (2005) increased or decreased by the estimated uncertainty of $25 \%$ (models VII and VIII of Table 1). As shown in Fig. 1, the effect is negligible for all the masses.

Thus, at variance with results published in the past, now the uncertainty in the ${ }^{12} \mathrm{C}+\alpha$ nuclear rate is noticeably reduced so that its effect on the loop morphology appears negligible. Consequently, in this paper, we neglect the uncertainty associated with this cross section in the evaluation of the error budget.

In conclusion, if one takes at their face value the present estimated uncertainties of the cross sections relevant for Cepheids star evolution, one finds that now the precision of the experimental measurements, at the energies of astrophysical interest, is high enough to rule out significant effects of these uncertainties on the loop extension and thus on the pulsational quantities.

\section{Effects of the chemical composition uncertainties}

In this section we present the effects of the LMC Cepheid chemical composition uncertainties on evolutionary properties such as the He burning loop extension and the $M L$ relation, and on pulsation properties such as the instability strip morphology and the $P L$ relations.

For the LMC iron content we adopt $[\mathrm{Fe} / \mathrm{H}]=-0.40$ that, as quoted above, is consistent with the estimates by Luck et al. (1998) and Romaniello et al. (2008). In particular Luck et al. (1998) suggest a range of $-0.55 \leq[\mathrm{Fe} / \mathrm{H}] \leq-0.19$. By adopting the Asplund et al. (2005) solar mixture, that is a $(Z / X)_{\odot}$ value of 0.0165 , from the assumed $[\mathrm{Fe} / \mathrm{H}]$ one obtains (see Eq. (2))
$Z=0.005$ (our "standard" value). On the basis of the observed range suggested by Luck et al. (1998) we allowed $Z$ to vary in the range $0.0035 \leq Z \leq 0.008$. Taking into account the current uncertainties on $\Delta Y / \Delta Z$ (see e.g. Pagel \& Portinari 1998; Gennaro et al. 2008), we allowed a variation of the original helium content from $Y=0.26$ to $Y=0.28$. With $Z=0.005, Y=0.26$ corresponds to $\Delta Y / \Delta Z=2$, while $Y=0.28$ implies $\Delta Y / \Delta Z=6$.

One should also take into account the uncertainty on the solar mixture. As already discussed, tridimensional hydrodynamical atmospheric models by Asplund et al. (2005) reduced the derived abundances of CNO and other heavy elements with respect to previous estimates (Grevesse \& Sauval 1998, hereafter GS98). GS98 already improved the mixture by Grevesse \& Noels (1993), widely adopted in the literature, mainly revising the $\mathrm{CNO}$ and $\mathrm{Ne}$ abundance and confirming the very good agreement between the new photospheric and meteoric results for the solar iron abundance.

The change of the adopted heavy element ratios might affect the evolutionary calculations of LMC Cepheids in two different ways: 1) changing the evolutionary characteristics at fixed metallicity; 2) changing the LMC inferred metallicity from the observed $[\mathrm{Fe} / \mathrm{H}]$. Regarding the second point, the adoption of the GS98 solar mixture leads to a LMC metallicity of $Z \approx 0.008$, which is within the previous quoted metallicity uncertainty. The first point is analysed by taking into account the effects on the evolutionary features of a solar mixture variation at fixed metallicity, as due to its effect on opacity, nuclear burning and equation of state calculations.

In the following we present different sets of models varying the chemical composition within the quoted ranges of uncertainty. The combinations of $Y$ and $Z$ values adopted for the different sets of models are summarized in Table 1. The first column of Table 1 gives the reference name of the treated cases (std, $Z_{\text {low }}, Z_{\text {high }}$ etc.), the second and the third columns give the adopted $Y$ and $Z$ values, whereas the fourth column reports the adopted chemical mixture at fixed $Z$. The cases XI, XII and XIII will be presented in detail in Sect. 6.1 in which we will discuss the effect of a mild core overshooting in the H-burning phase.

For each case of Table 1 we computed evolutionary and nonlinear convective pulsation models spanning a stellar mass range from 3-4 $M_{\odot}$ to $13-14 M_{\odot}$, which covers the range of masses crossing the instability strip (see for details Sect. 5.3).

All the computed sets for the cases I to VI of Table 1 are plotted in Fig. 4; the fits of the corresponding instability strips calculated as explained in Sect. 5.2 are superimposed on the evolutionary models.

Some models show a smaller secondary loop during the redward excursion in the central He burning phase (see e.g. Bono et al. 2000; Salasnich et al. 1999, too), which is not a numerical artifact. The physical reason for this secondary loop is a slight increase in the size of the convective core, due to the opacity increase in the $\mathrm{C}$ and $\mathrm{O}$ rich nucleus, leading to small variations of the $\mathrm{H}$ and He burning efficiency. The mechanism is the ana$\log$ of the breathing pulses occurring in low mass stars (see e.g. Sweigart \& Demarque 1972, 1973; Castellani et al. 1985).

\subsection{Loop extension}

Figure 2 shows, for a $7 M_{\odot}$ model, the effects on the blue loop extension of variations in the helium and metal contents within the uncertainties of these quantities (cases from I to IV of Table 1). The upper panel shows that increasing the metal content, at fixed helium abundance, the extension of the blue loop decreases, whereas the lower panel shows that an increase of the helium 
Table 3. The same as Table 2 but for quadratic fits.

\begin{tabular}{lllll}
\hline \hline Case & $a$ & $b$ & $c$ & $\sigma$ \\
\hline Fundamental blue edge & & & & \\
I $($ std $)$ & $3.744 \pm 0.020$ & $0.039 \pm 0.012$ & $-0.009 \pm 0.002$ & 0.002 \\
II $\left(Z_{\text {low }}\right)$ & $3.751 \pm 0.058$ & $0.028 \pm 0.032$ & $-0.006 \pm 0.004$ & 0.005 \\
III $\left(Z_{\text {high }}\right)$ & $3.594 \pm 0.060$ & $0.137 \pm 0.037$ & $-0.024 \pm 0.005$ & 0.005 \\
IV $\left(Y_{\text {high }}\right)$ & $3.672 \pm 0.032$ & $0.086 \pm 0.018$ & $-0.016 \pm 0.003$ & 0.003 \\
V $\left(Y_{\text {high }}, Z_{\text {high }}\right)$ & $3.748 \pm 0.104$ & $0.046 \pm 0.063$ & $-0.011 \pm 0.009$ & 0.008 \\
VI $\left(Z_{\text {high }}, G S 98\right)$ & $3.599 \pm 0.076$ & $0.131 \pm 0.046$ & $-0.023 \pm 0.007$ & 0.007 \\
Fundamental red edge & & & & \\
I $(s t d)$ & $3.878 \pm 0.112$ & $-0.028 \pm 0.064$ & $-0.006 \pm 0.009$ & 0.010 \\
II $\left(Z_{\text {low }}\right)$ & $4.071 \pm 0.261$ & $-0.146 \pm 0.147$ & $0.012 \pm 0.020$ & 0.025 \\
III $\left(Z_{\text {high }}\right)$ & $3.728 \pm 0.059$ & $0.071 \pm 0.036$ & $-0.023 \pm 0.005$ & 0.004 \\
IV $\left(Y_{\text {high }}\right)$ & $3.988 \pm 0.118$ & $-0.098 \pm 0.068$ & $0.004 \pm 0.009$ & 0.010 \\
V $\left(Y_{\text {high }}, Z_{\text {high }}\right)$ & $3.788 \pm 0.032$ & $0.031 \pm 0.019$ & $-0.016 \pm 0.003$ & 0.002 \\
VI $\left(Z_{\text {high }}, G S 98\right)$ & $3.731 \pm 0.031$ & $0.064 \pm 0.019$ & $-0.020 \pm 0.003$ & 0.003 \\
\hline & & & &
\end{tabular}
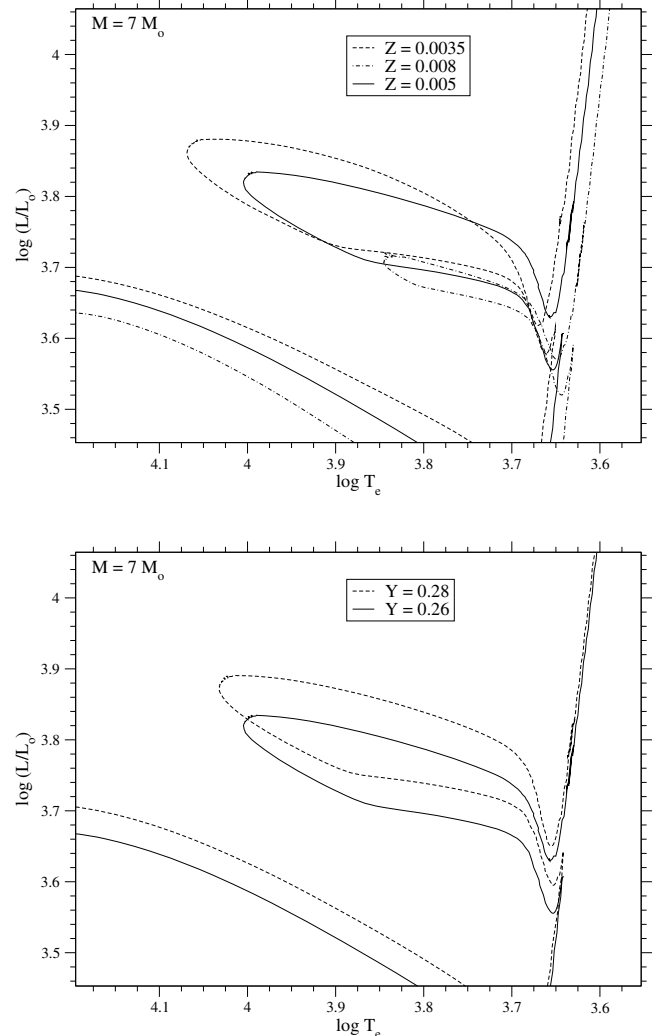

Fig. 2. Upper panel: effect of a metallicity variation on the loop extension for a $7 M_{\odot}$; the three different lines correspond to cases I, II, III of Table 1: std case $\left(Z=0.005\right.$, solid line) $Z_{\text {low }}=0.0035$ (dashed line) and $Z_{\text {high }}=0.008$ (dot-dashed line). Lower panel: as in the upper panel but for a helium abundance variation and for cases I ( $s t d, Y=0.26$, solid line $)$ and IV ( $Y_{\text {high }}=0.28$, dashed line $)$ of Table 1.

content within its uncertainty range, at fixed metallicity, leads to more extended loops, even if the effect is reduced compared to that of lowering $Z$. The behaviour is similar for the other analysed model masses.

A simultaneous variation of $Z$ and $Y$ within their uncertainty ranges does not result in an additive effect on the temperature extension of the loop. An example of this behaviour is shown in Fig. 4f, for which the set of evolutionary tracks was computed
Table 4. The same as Table 2 but for the first overtone boundaries.

\begin{tabular}{llcl}
\hline \hline Case & $a$ & $b$ & $\sigma$ \\
\hline First overtone blue edge & & & \\
I $(s t d)$ & $3.939 \pm 0.019-0.043 \pm 0.006$ & 0.005 \\
II $\left(Z_{\text {low }}\right)$ & $3.942 \pm 0.019-0.044 \pm 0.006$ & 0.005 \\
III $\left(Z_{\text {high }}\right)$ & $3.913 \pm 0.046-0.038 \pm 0.015$ & 0.013 \\
IV $\left(Y_{\text {high }}\right)$ & $3.922 \pm 0.027-0.038 \pm 0.008$ & 0.007 \\
V $\left(Y_{\text {high }}, Z_{\text {high }}\right)$ & $3.954 \pm 0.012-0.050 \pm 0.004$ & 0.003 \\
VI $\left(Z_{\text {high }}\right.$, GS98 $)$ & $3.962 \pm 0.012-0.052 \pm 0.004$ & 0.005 \\
First overtone red edge & & & \\
I $(s t d)$ & $3.847 \pm 0.010-0.023 \pm 0.003$ & 0.003 \\
II $\left(Z_{\text {low }}\right)$ & $3.834 \pm 0.004-0.018 \pm 0.001$ & 0.001 \\
III $\left(Z_{\text {high }}\right)$ & $3.840 \pm 0.009-0.024 \pm 0.003$ & 0.003 \\
IV $\left(Y_{\text {high }}\right)$ & $3.834 \pm 0.004-0.018 \pm 0.001$ & 0.001 \\
V $\left(Y_{\text {high }}, Z_{\text {high }}\right)$ & $3.826 \pm 0.004-0.018 \pm 0.001$ & 0.001 \\
VI $\left(Z_{\text {high }}, G S 98\right)$ & $3.831 \pm 0.014-0.021 \pm 0.005$ & 0.006 \\
\hline
\end{tabular}

with the upper values of $Y$ and $Z$, namely $Y=0.28$ and $Z=$ 0.008 (case $\mathrm{V}$ of Table 1). Beside the apparent non additivity in $\log T_{\mathrm{e}}$, there is rather complicated mass dependence with a more regular trend for the intermediate mass values $\left(\simeq 5-8 M_{\odot}\right)$ and an inverted trend for higher masses. Thus one should be very careful to predict the extension of the loop when more than one parameter is varied, as it cannot be simply evaluated by combining the results obtained for the variation of each single parameter separately.

The effect of changing the mixture at fixed $Z$ is shown by comparing Fig. 4c (case III of Table 1), in which the set of tracks is computed with $Z=0.008$ and the mixture of AGS05, with Fig. 4e (case VI of Table 1), computed with the same total metallicity $(Z=0.008)$ but with the GS98 mixture. To analyse this case we changed the original element abundances of our models and we calculated suitable opacity tables making advantage of the codes available at the OPAL web site ${ }^{4}$; the EOS is also varied following the mixture change (see Degl'Innocenti et al. 2006 , for a more detailed analysis of the effects of a mixture update on the stellar models).

${ }^{4}$ http://www-phys.llnl .gov/Research/OPAL/opal.html 

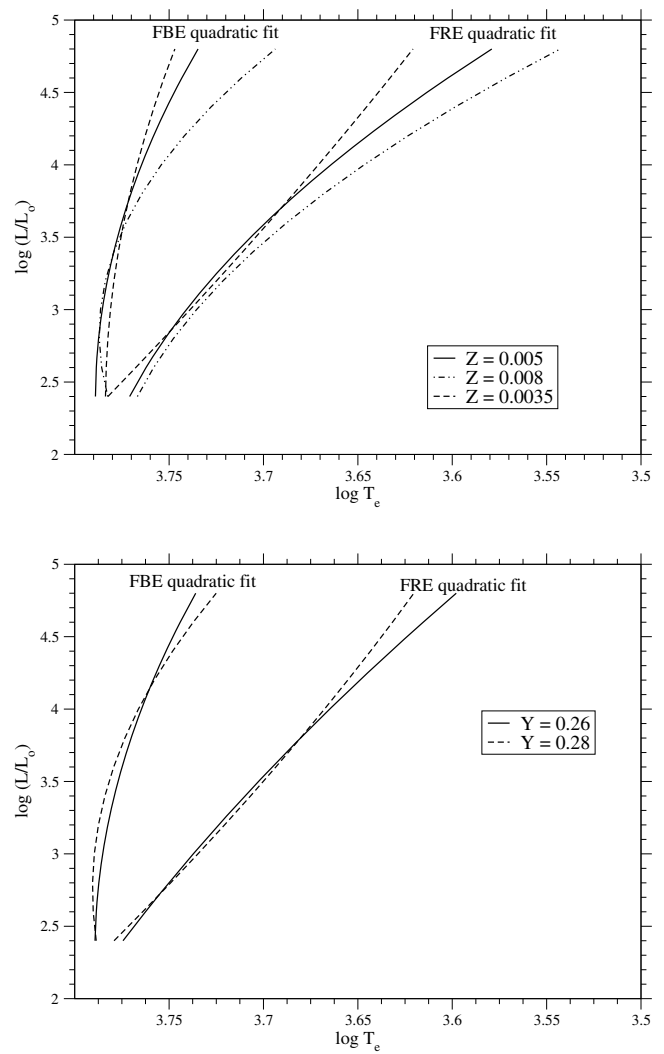

Fig. 3. Upper panel: effect on the fundamental instability strip edges of a metallicity variation within its uncertainty (cases I, II, III of Table 1). Lower panel: as in the upper panel but for a variation of the original helium abundance (cases I, IV of Table 1). In both panels the quadratic fits corresponding to Table 3 are shown.

The mixture change at fixed $Z$ has a negligible effect on the loop extension for $M \leq 8 M_{\odot}$. For higher masses we find that models with Asplund et al. (2005) composition do not show blue loops at all, whereas the ones with the Grevesse \& Sauval (1998) mixture display extended blue loops. As this effect is evident only for about half of the analyzed mass range we decided to neglect it in the following.

\subsection{Instability strip}

For each chemical composition, mass and luminosity, the modal stability of pulsation models has been investigated for both the fundamental and the first overtone mode. The effective temperatures (hereafter in $\mathrm{K}$ ) of the hottest and coolest pulsating fundamental models, increased and decreased, respectively, by the extension of the pass in temperature adopted in our analysis, $50 \mathrm{~K}$, correspond to the fundamental blue and red edges (FBE and FRE). Similarly the first overtone blue (FOBE) and red (FORE) edges are evaluated. Table 3 reports the quadratic fit coefficients of the FBE and FRE, plotted in Fig. 4 as solid lines. The linear fits of the FBE and FRE are available on line in Table 2, whereas the linear fits for the FOBE and FORE, plotted in Fig. 4 as dashed lines, are reported in Table 4 in the form $\log T_{\mathrm{e}}=a+b \log \left(L / L_{\odot}\right)$, where $\sigma$ is the least square estimate of the intrinsic dispersion. Figures $4 \mathrm{a}-\mathrm{f}$ show the evolutionary tracks superimposed with the quadratic fits of the edges of the fundamental mode instability strips and the linear fits of the first overtone ones for the cases I, II, III, IV, V, VI of Table 1.
Table 5. Maximum $\left(M_{\max }\right)$ and minimum $\left(M_{\min }\right)$ mass entering the instability strip, for the various model sets.

\begin{tabular}{lll}
\hline \hline Case & $M_{\min }\left(M_{\odot}\right)$ & $M_{\max }\left(M_{\odot}\right)$ \\
\hline I $(s t d)$ & 4 & 12 \\
II $\left(Z_{\text {low }}\right)$ & 3 & 14 \\
III $\left(Z_{\text {high }}\right)$ & 4 & 8 \\
IV $\left(Y_{\text {high }}\right)$ & 4 & 13 \\
\hline
\end{tabular}

To analyse the effect of the chemical composition uncertainty, the upper panel of Fig. 3 shows the instability strips computed for the different metallicities corresponding to the cases I, II, III of Table 1; whereas in the lower panel of the same figure the effect on the instability strip of the $Y$ variation within its uncertainty is shown (cases I and IV of Table 1). As already found in our previous papers (Bono et al. 1999b; Caputo et al. 2000) the predicted instability strip becomes redder as the metallicity increases, while increasing the helium content from 0.26 to 0.28 at $Z=0.005$ only produces a slight narrowing of the instability strip at the highest luminosity levels, likely due to the slightly reduced efficiency of the $\mathrm{H}$ ionization region in driving pulsation.

\subsection{The mass-luminosity relation}

Inspection of Fig. 4 suggests that the loop extension generally increases with increasing mass, but, in some cases, it decreases for the $5 M_{\odot}$ model. As discussed by Castellani et al. (1990) this is because for masses around this value the first dredge-up (which is active for lower masses) becomes inefficient and the star reaches the central He burning phase with a reduced external He abundance (and thus with a reduced loop extension) with respect to the lower masses.

For each model set we found the minimum $\left(M_{\min }\right)$ and maximum $\left(M_{\max }\right)$ mass which cross the instability strip. The $M_{\max }$ and $M_{\min }$ values are listed in Table 5, except for the cases including overshooting. The minimum mass in general increases with the metallicity and the helium content while the maximum mass increases for a decrease of the metallicity and an increase of $Y$.

To derive the $M L$ relation (see e.g. Bono et al. 2000) we take into account the three crossings of the instability strip: the first crossing during the $\mathrm{H}$ burning and the second and third crossings during the He burning phases. The mean luminosity is obtained by averaging the luminosity behaviour during the three subsequent crossings with the corresponding evolutionary times. Following this procedure, for each case from I to IV of Table 1 we derived the corresponding $M L$ relation (the results are available on line in Table 6).

Since one of the purposes of this work is to investigate the spread of the $M L$ relation due to uncertainties on the chemical composition, we calculated the global $M L$ relation by fitting together all the $M L$ data sets (cases I-IV of Table 1). As a result we find:

$\log \left(L / L_{\odot}\right)=1.015( \pm 0.021)+3.150( \pm 0.024) \log \left(M / M_{\odot}\right)$

with a standard deviation of $\sigma=0.025$; the errors include the effect of varying the chemical abundances among the different cases. The comparison with the $M L$ relations obtained for the individual cases separately (see Table 6 on line) suggest that the coefficients of the global $M L$ relation are consistent with 

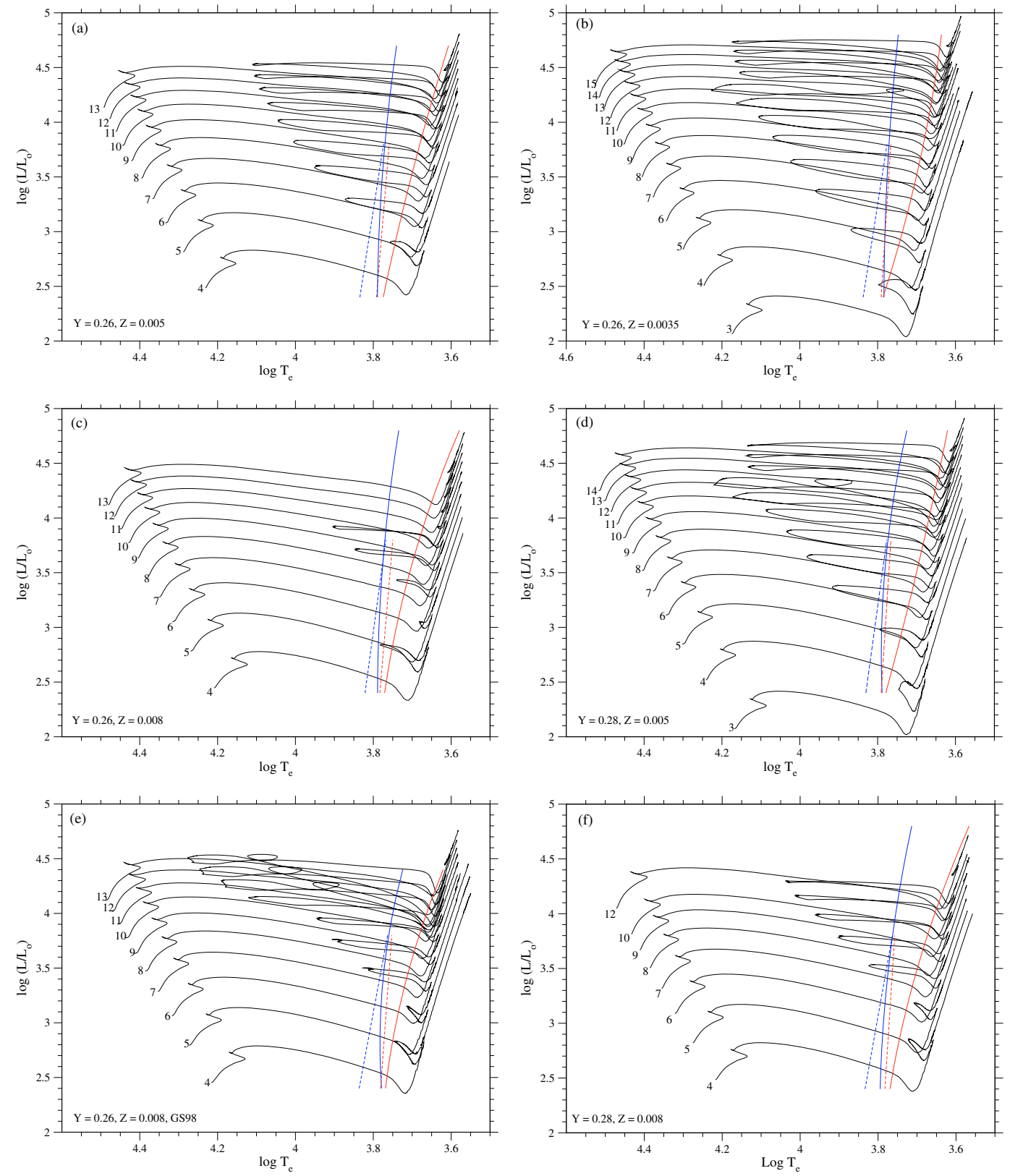

Fig. 4. Panels a) to f): evolutionary tracks, quadratic fits of the fundamental mode instability strip boundaries and linear fits of the first overtone ones, for the cases I to VI of Table 1.

the ones of the individual relations apart from case III $\left(Z_{\text {high }}\right)$ that corresponds to a significantly steeper relation, with a fainter zero-point, than all the others. This occurrence produces an increase of the intrinsic dispersion when passing from the individual $M L$ relations to the global one.

To compare the present result with a previous published relation based on evolutionary and pulsation models including older physical inputs, we compared the present $M L$ relation, given by Eq. (3), with the one by Bono et al. (2000), which explicitly takes into account the dependence on the chemical composition, computed for the values of $Y$ and $Z$ corresponding to our standard case: $Y=0.26, Z=0.005$. We found that the $M L$ relation derived in this paper is flatter than Bono et al. (2000) with the disagreement reaching about $0.1 \mathrm{dex}$ at masses around $10 M_{\odot}$. However for $M \leq \sim 8 M_{\odot}$ the two relations are consistent within the uncertainties.

\subsection{Period-luminosity relations}

For the cases I to IV of Table 1 we calculated the $P L T_{\mathrm{e}}$ relations for the fundamental and first overtone models; the tables with the coefficients for these relations are available on line in Table 7.

We derived the synthetic $P L$ relations by populating the predicted instability strip according to an assumed mass distribution and adopting, for each mass and chemical composition, the corresponding $M L$ relation derived on the basis of the corresponding set of evolutionary tracks (see Sect. 5.3). More in detail, for each treated case (I to IV of Table 1), we built a synthetic population by extracting masses according to a Salpeter initial mass function (IMF) $\left(\propto M^{-2.35}\right)$. The range of generated masses is given by the values of $M_{\max }$ and $M_{\min }$ for the cases I to IV listed in Table 5. In agreement with the procedure adopted in previous papers (Caputo et al. 2000; Kennicutt et al. 1998), 
Table 8. Maximum $\left(P_{\max }\right)$ minimum $\left(P_{\min }\right)$ periods in days derived from the synthetic Cepheid distributions.

\begin{tabular}{lll}
\hline \hline Case & $P_{\min }$ (days) & $P_{\max }$ (days) \\
\hline I $($ std $)$ & 2.5 & 72.9 \\
II $\left(Z_{\text {low }}\right)$ & 1.4 & 87.6 \\
III $\left(Z_{\text {high }}\right)$ & 2.1 & 21.9 \\
IV $\left(Y_{\text {high }}\right)$ & 2.6 & 92.0 \\
\hline
\end{tabular}

we fixed to $N=1000$ the total number of extractions falling into the instability strip. To each synthetic star we assigned the luminosity derived from the corresponding $M L$ relation. Once the luminosity is fixed, from the quadratic fits of the fundamental edges, derived in Sect. 5.2, we obtained the corresponding temperature of the FRE and the FBE. In the temperature range from the $T_{\mathrm{e}}$ of the FBE (decreased by $50 \mathrm{~K}$ ) to the $T_{\mathrm{e}}$ of the FRE (increased by $50 \mathrm{~K}$ ), we then extracted randomly the temperature $T_{\mathrm{e}}$ of the synthetic star. For each extracted $T_{\mathrm{e}}$ (in $\mathrm{K}$ ) the period (in days) is obtained from the $P=f\left(L, M, T_{\mathrm{e}}\right)$ relation, resulting from a linear regression through the fundamental models of each selected case. Then, by means of the static model atmospheres by Bessel et al. (1998), for the explored chemical compositions, we converted the model intrinsic luminosities into the various photometric bands, obtaining synthetic distributions in periodmagnitude planes. The $P L$ relations are derived by means of linear (or quadratic) regressions through these model distributions.

The maximum and minimum periods corresponding to the obtained synthetic distributions are reported in Table 8. In Tables 9 and 11 we report the linear $\left(\overline{M_{\lambda}}=a+b \log P\right)$ and quadratic $\left(\overline{M_{\lambda}}=a+b \log P+c(\log P)^{2}\right)$ period-luminosity relations for fundamental pulsators in the different photometric bands. In these Tables we listed the fit coefficients for case I of Table 1 (std) and the global fit of cases I to IV taken together (all), as well as the results for the $P L$ relations obtained by rejecting periods longer than $\log P \geq 1.5$ (cut). The case all provides an estimate of the effect of the uncertainties on the chemical abundances on the predicted $P L$ relations. The linear and quadratic $P L_{\lambda}$ relations, computed for the single cases II, III and IV of Table 1, are available on line in Tables 10 and 12.

Passing from the individual cases to the global $P L$ relations, the coefficients and the intrinsic dispersion do not vary significantly because the effects of these variations in the abundances are smaller than the effect on the intrinsic scatter due to the finite width of the instability strip, especially in the optical filters. Moreover, inspection of Table 9 suggests that the $P L$ relations become more linear, less dependent on the chemical composition and with a smaller intrinsic dispersion passing from the optical to the near-infrared filters, confirming previous observational (Madore \& Freedman 1991) and theoretical (Bono et al. 1999b; Caputo et al. 2000) results. The $P L$ relations obtained for $\log P<1.5$ are derived because, as shown in Caputo et al. (2000), removing the longest periods, the period-magnitude distribution is better reproduced by a linear relation also in the optical bands. Moreover, many observed Cepheid samples cover this period range.

The deviation from linearity of Cepheid $P L$ relations is a debated issue (Sandage et al. 2004; Kanbur et al. 2004; Ngeow et al. 2005; Kanbur et al. 2006) and for this reason we also computed quadratic $P L$ relations. In particular, Ngeow et al. (2005) performed a statistical investigation of the LMC classical Cepheid sample obtained from the MACHO database and found that the observed behaviour in period-magnitude diagrams is best reproduced by two linear relations, with a break
Table 9. Theoretical linear period-luminosity relations in different photometric bands for fundamental pulsators in the standard and global cases.

\begin{tabular}{llll}
\hline \hline Case & $a$ & $b$ & $\sigma$ \\
\hline & & $\overline{M_{B}}$ & \\
std & $-1.04 \pm 0.03$ & $-2.30 \pm 0.03$ & 0.32 \\
all & $-0.87 \pm 0.01$ & $-2.43 \pm 0.01$ & 0.32 \\
std cut & $-0.95 \pm 0.03$ & $-2.42 \pm 0.03$ & 0.30 \\
all cut & $-0.83 \pm 0.01$ & $-2.49 \pm 0.02$ & 0.30 \\
& & $\overline{M_{V}}$ & \\
std & $-1.40 \pm 0.02$ & $-2.69 \pm 0.02$ & 0.23 \\
all & $-1.28 \pm 0.01$ & $-2.77 \pm 0.01$ & 0.23 \\
std cut & $-1.34 \pm 0.02$ & $-2.77 \pm 0.02$ & 0.22 \\
all cut & $-1.25 \pm 0.01$ & $-2.82 \pm 0.01$ & 0.22 \\
& & $\overline{M_{R}}$ & \\
std & $-1.65 \pm 0.02$ & $-2.83 \pm 0.02$ & 0.20 \\
all & $-1.55 \pm 0.01$ & $-2.91 \pm 0.01$ & 0.20 \\
std cut & $-1.59 \pm 0.02$ & $-2.91 \pm 0.02$ & 0.18 \\
all cut & $-1.52 \pm 0.01$ & $-2.95 \pm 0.01$ & 0.19 \\
& & $\overline{M_{I}}$ & \\
std & $-1.92 \pm 0.01$ & $-2.96 \pm 0.02$ & 0.17 \\
all & $-1.84 \pm 0.01$ & $-3.01 \pm 0.01$ & 0.17 \\
std cut & $-1.87 \pm 0.02$ & $-3.02 \pm 0.02$ & 0.15 \\
all cut & $-1.82 \pm 0.01$ & $-3.05 \pm 0.01$ & 0.16 \\
& & $\overline{M_{J}}$ & \\
std & $-2.238 \pm 0.010$ & $-3.149 \pm 0.011$ & 0.12 \\
all & $-2.184 \pm 0.005$ & $-3.184 \pm 0.005$ & 0.12 \\
std cut & $-2.206 \pm 0.011$ & $-3.191 \pm 0.012$ & 0.11 \\
all cut & $-2.166 \pm 0.005$ & $-3.210 \pm 0.006$ & 0.11 \\
& & $\overline{M_{K}}$ & \\
std & $-2.499 \pm 0.006$ & $-3.329 \pm 0.006$ & 0.07 \\
all & $-2.470 \pm 0.003$ & $-3.343 \pm 0.003$ & 0.08 \\
std cut & $-2.481 \pm 0.007$ & $-3.353 \pm 0.008$ & 0.07 \\
all cut & $-2.459 \pm 0.003$ & $-3.359 \pm 0.004$ & 0.07 \\
\hline & & &
\end{tabular}

at 10 days. Nonlinear pulsation models (see also Caputo et al. 2000; Fiorentino et al. 2002; Marconi et al. 2005) suggest a quadratic form of $P L$ relations, in particular in the optical bands (see Table 11). However the effect of this nonlinearity on the calibration of the extragalactic distance scale is very small (see Koen et al. 2007). In Fig. 5 we plotted the predicted synthetic $P L$ relations in the V (upper panel) and I (lower panel) JohnsonCousins photometric bands with the quadratic fits superimposed (solid lines) for cases I to IV taken together (case all in Table 11). As a comparison the two linear regressions (for $\log P<1.0$ and $\log P \geq 1.0$, dashed lines) obtained observationally by Sandage et al. (2004) are also shown. In panel a of Fig. 6 we show the residuals of the present linear $P L$ relations for $\log P<1.5$ (case all cut of Table 9) in the $V, I$ bands and over the whole period range for the $K$ band with respect to the empirical relations by Freedman et al. (2001) (for $V$ and $I$ ) and Persson et al. (2004) (for the $K$ band). According to this plot, we find differences smaller than $0.2 \mathrm{mag}$ (in absolute value) in all the bands. Panel b of Fig. 6 shows the differences between the present linear solutions with $\log P<1.5$ and the theoretical $P L$ linear relations with $\log P<1.5$ by Caputo et al. (2000). Again we find differences within 0.2 mag with an opposite trend as a function of the period with respect to the previous comparison, thus suggesting that the the magnitudes predicted by the new $P L$ relations obtained in this paper are somewhat intermediate between the ones based on empirical relations and the theoretical relations by Caputo et al. (2000).

As far the physical assumptions of our pulsation models are concerned, we have already shown that changes in the adopted 

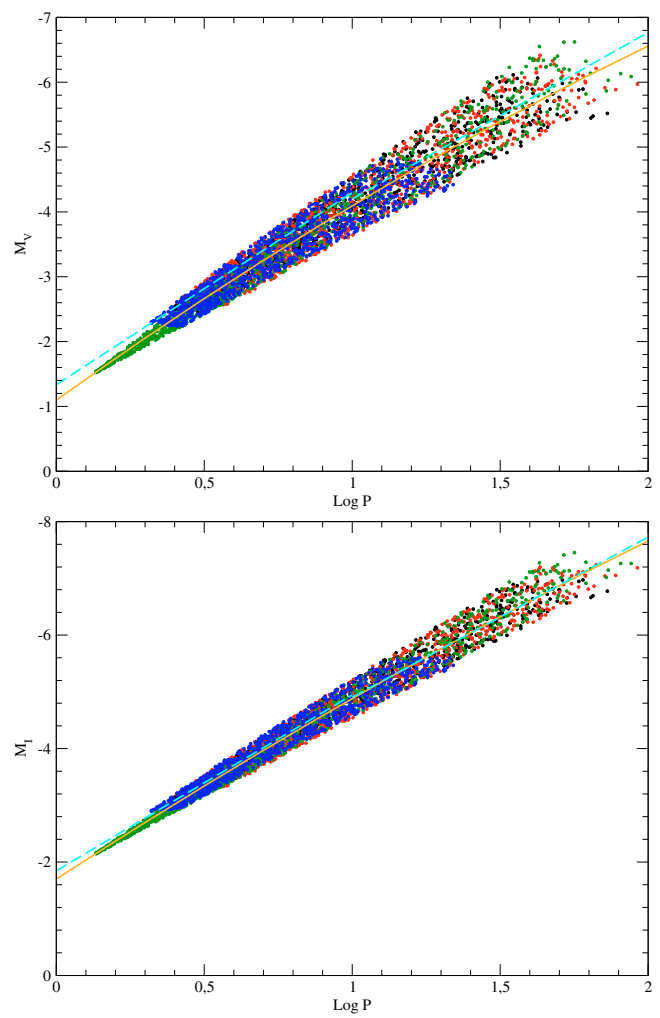

Fig. 5. Synthetic period-luminosity relations in the $V$ (upper panel) and $I$ (lower panel) Johnson-Cousins photometric bands with the quadratic fits (solid lines) corresponding to the case all of Table 11. Symbols: std case (case I, black dots); $Z_{\text {low }}$ (case II, green dots); $Z_{\text {high }}$ (case III, blue dots); $Y_{\text {high }}$ (case IV, red dots).

equation of state do not affect significantly the pulsation scenario (Petroni 2003). On the other hand, variations of the mixing length parameters used to close the nonlinear equation system (see Bono \& Stellingwerf 1994) have been found to produce negligible effects on the predicted Cepheid relations (see Fiorentino et al. 2007, for details).

\section{Noncanonical models}

\subsection{Overshooting}

The extension of the mixing into a region that is stable following the Schwarzschild criterion is generally called "overshooting" (see e.g. Cordier et al. 2002, and references therein). An additional extension of the canonical convective regions could also be due to rotationally induced mixing (see e.g. Meynet \& Maeder 2000); both phenomena can be modeled, at least as far as the extension of the mixing zones is concerned, with the same "overshooting" formalism. A complete understanding of these extra-mixing mechanisms is still lacking in the literature due to the complexity of the involved phenomena, that also prevents an analytical approach (see e.g. Straka et al. 2005; Demarque et al. 2007). Three-dimensional hydrodynamical calculations are still under development (Eggleton et al. 2007; Meakin \& Arnett 2007). Thus a semi-empirical approach is currently used by defining the extension of any additional mixing region, starting from the border of the canonical convective zones, in terms of the pressure scale height $H_{\mathrm{p}}: l_{\mathrm{ov}}=\beta H_{\mathrm{p}}$. The overshooting efficiency is generally estimated through the comparison between theory and observations for different relevant quantities, and is
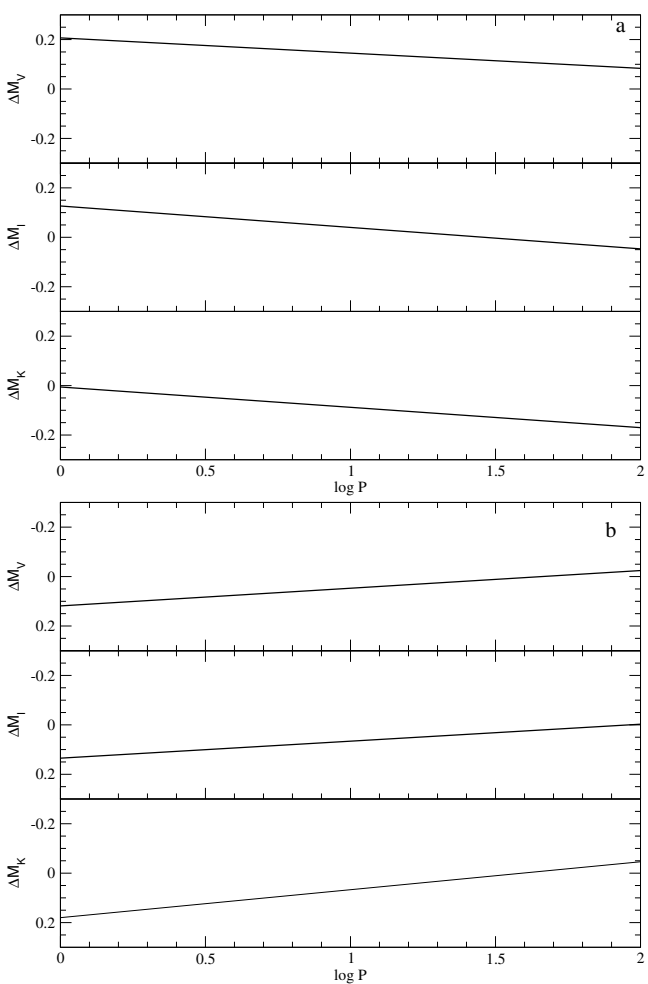

Fig. 6. a) Residuals of the present linear $P L$ relations for $\log P<1.5$ in the $V, I$ bands and over the whole period range for the $K$ band with respect to the empirical relations; b) Differences between the present linear solutions with $\log P<1.5$ and the theoretical ones by Caputo et al. (2000).

still widely debated point (see e.g. Claret 2007; Brocato et al. 2003; Barmina et al. 2002; Chiosi et al. 1992, and references therein).

We calculated additional sets of evolutionary models including overshooting during the central $\mathrm{H}$ burning phase (Main Sequence, MS) with $l_{\mathrm{ov}}=0.1 H_{\mathrm{p}}$ and $l_{\mathrm{ov}}=0.25 H_{\mathrm{p}}$, within the range suggested in the recent literature (see e.g. Girardi et al. 2000). During the central He burning phase our code implements the classical semi-convection criterion (see e.g. Castellani et al. 1985) at the border of the Schwarzschild convective core (which is driven by mechanical overshooting at the convective core boundary), while breathing pulses (see e.g. Castellani et al. 1985; Dorman \& Rood 1993) are suppressed following the procedure described by Caputo et al. (1989). In the present models the overshooting region is fully homogenized but the temperature gradient is kept at its radiative value.

The inclusion of overshooting during central $\mathrm{H}$ burning in standard models leads to a higher luminosity and longer lifetime during the MS phase, larger He cores and luminosities and reduced lifetimes during the following central He burning phase. This reflects in the adoption of a different mass-luminosity relation for Cepheid pulsational models. The adopted luminosity levels for noncanonical models in this paper are taken as brighter than the canonical ones by $0.25 \mathrm{dex}$, following the prescriptions by Chiosi et al. (1993). We checked that it is true for current evolutionary models too.

When the overshooting phenomenon is included, the blue loops in the color-magnitude diagram are less extended than in the absence of overshooting in such a way that for some chemical compositions intermediate-mass models do not cross the classical Cepheid instability strip. To avoid this problem, some 
Table 11. Theoretical quadratic period-luminosity relations for fundamental pulsators in the standard and global cases.

\begin{tabular}{lllll}
\hline \hline Case & $a$ & $b$ & $c$ & $\sigma$ \\
\hline & & $\overline{M_{B}}$ & & \\
std & $-0.43 \pm 0.08$ & $-3.69 \pm 0.17$ & $0.69 \pm 0.08$ & 0.31 \\
all & $-0.63 \pm 0.02$ & $\frac{-3.07 \pm 0.06}{M_{V}}$ & $0.35 \pm 0.03$ & 0.32 \\
& & & \\
std & $-0.98 \pm 0.06$ & $-3.66 \pm 0.12$ & $0.48 \pm 0.06$ & 0.22 \\
all & $-1.10 \pm 0.02$ & $-3.27 \pm 0.04$ & $0.27 \pm 0.02$ & 0.23 \\
& & $\overline{M_{R}}$ & & \\
std & $-1.29 \pm 0.05$ & $-3.65 \pm 0.10$ & $0.41 \pm 0.05$ & 0.19 \\
all & $-1.39 \pm 0.02$ & $-3.33 \pm 0.04$ & $0.23 \pm 0.02$ & 0.19 \\
& & $\overline{M_{I}}$ & & \\
std & $-1.61 \pm 0.04$ & $-3.66 \pm 0.09$ & $0.35 \pm 0.04$ & 0.16 \\
all & $-1.70 \pm 0.01$ & $-3.38 \pm 0.03$ & $0.20 \pm 0.02$ & 0.16 \\
& & $\overline{M_{J}}$ & & \\
std & $-2.03 \pm 0.03$ & $-3.63 \pm 0.06$ & $0.24 \pm 0.03$ & 0.12 \\
all & $-2.08 \pm 0.01$ & $-3.45 \pm 0.02$ & $0.14 \pm 0.01$ & 0.12 \\
& & $\overline{M_{K}}$ & & \\
std & $-2.38 \pm 0.02$ & $-3.61 \pm 0.04$ & $0.14 \pm 0.02$ & 0.07 \\
all & $-2.41 \pm 0.01$ & $-3.50 \pm 0.01$ & $0.09 \pm 0.01$ & 0.07 \\
\hline
\end{tabular}

Table 13. Quadratic analytical relations for the fundamental edges, in the non canonical standard case.

\begin{tabular}{|c|c|c|c|}
\hline $\bar{a}$ & $b$ & $\bar{c}$ & $\bar{\sigma}$ \\
\hline $3.904 \pm 0.046$ & $\begin{array}{l}\text { Blue edge } \\
-0.034 \pm 0.027 \\
\text { Red edge }\end{array}$ & $-0.0001 \pm 0.0038$ & 0.002 \\
\hline $3.816 \pm 0.078$ & $0.008 \pm 0.046$ & $-0.0124 \pm 0.0065$ & 0.004 \\
\hline
\end{tabular}

authors include an "ad hoc" undershooting at the lower boundary of convective envelopes (see Girardi et al. 2000), even if there is no clear observational evidence for this mechanism (see e.g. Renzini \& Ritossa 1994; Riello et al. 2003).

Relevant pulsation properties, such as the morphology and the amplitude of light and radial velocity curves, are affected by the assumed $M L$ relation (Bono et al. 2002; Natale et al. 2008), and, in turn, by the amount of overshooting accounted for in the models (Caputo et al. 2005; Keller 2008, and references therein). Independent theoretical and observational evidence suggests that the Cepheid pulsation masses are lower than the canonical evolutionary values but the amount of this discrepancy is not firmly established even if several authors find a value of the order of 10-15\%. (e.g. Bono et al. 2001; Beaulieu et al. 2001; Caputo et al. 2005; Natale et al. 2008, and references therein).

To quantify the effect of overshooting, we computed models with standard chemical composition $(Z=0.005, Y=0.26)$ for two selected values of the overshooting efficiency $(\beta=0.1$ and $\beta=0.25$, models XI and XIII of Table 1$)$. However, due to the reduced loop extension, some intermediate mass models with $\beta=0.25$ do not cross the instability strip, in disagreement with observations of LMC Cepheids in this region. To avoid this problem without including overshooting for the convective envelopes, taking into account the present uncertainty in the original helium abundance, we calculated overshooting models for $Z=0.005, Y=0.28$ (model XII of Table 1 ) which, as already discussed, show a more extended loop than the standard models. In this way, even models with $\beta=0.25$ populate the instability strip.

In Table 13 the coefficients of the quadratic fits of the FRE and FBE for models with $l_{\mathrm{ov}}=0.25 H_{\mathrm{p}}$ and standard chemical composition are reported.
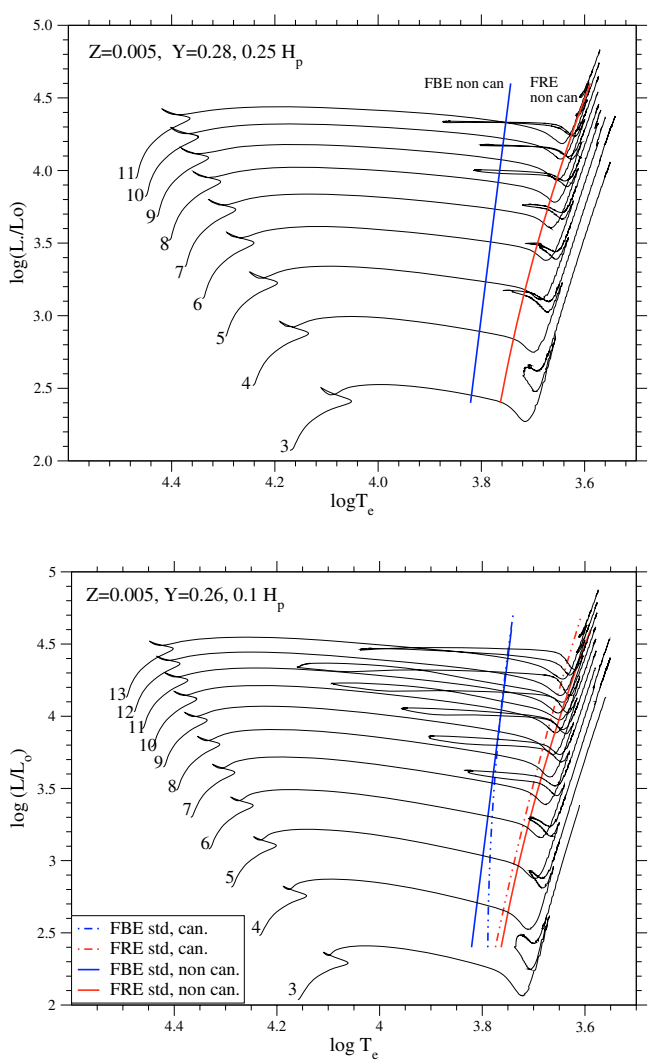

Fig. 7. Evolutionary tracks with $Y=0.28, Z=0.005, l_{\mathrm{ov}}=0.25 H_{\mathrm{p}}$ and the quadratic fit of the noncanonical instability strip boundaries (upper panel); evolutionary tracks for $Y=0.26, Z=0.005, l_{\mathrm{ov}}=0.1 H_{\mathrm{p}}$ and the quadratic fit of the canonical (dot-dashed lines) and noncanonical (solid lines) instability strip boundaries for standard chemical composition (lower panel).

The upper panel of Fig. 7 shows the evolutionary tracks with $l_{\mathrm{ov}}=0.25 H_{\mathrm{p}}, Z=0.005, Y=0.28$ and the quadratic fit of the non canonical strip, while the lower panel of the same figure shows the evolutionary tracks with $l_{\mathrm{ov}}=0.1 H_{\mathrm{p}}, Z=0.005, Y=$ 0.26 and the quadratic fits of the canonical (dot-dashed lines) and non canonical (solid lines) instability strip boundaries calculated for standard chemical composition. We notice that the case with $\beta=0.1$ is not significantly different from the canonical one and for this reason in the following we will concentrate on the case with $\beta=0.25$.

The $M L$ obtained with the set of tracks with $l_{\mathrm{ov}}=0.25 H_{\mathrm{p}}$, $Z=0.005, Y=0.28$ combined with the noncanonical strip of Fig. 7, upper panel, is the following:

$\log \left(L / L_{\odot}\right)=1.260( \pm 0.061)+3.187( \pm 0.076) \log \left(M / M_{\odot}\right)$

with $\sigma=0.022$. As expected this $M L$ relation has a brighter zero-point than the canonical one (see Eq. (3)) by about $0.25 \mathrm{dex}$, and the same slope within the errors. This difference can be also noted from the comparison shown in Fig. 8.

For the chemical composition of case I, in Table 1 we calculated the $P L T_{\mathrm{e}}$ relations for noncanonical fundamental and first overtone models; the tables with the coefficients for these relations are available on line in Table 14.

Adopting the same procedure as for canonical models we derived the multi-band period-luminosity relations for $\beta=0.25$, $Z=0.005, Y=0.28$. The coefficients of the linear $P L$ in the $V, I, K$ bands are reported in Table 17, whereas the linear and quadratic $P L$ relations in the other photometric bands are available on line, in Tables 15 and 16. 


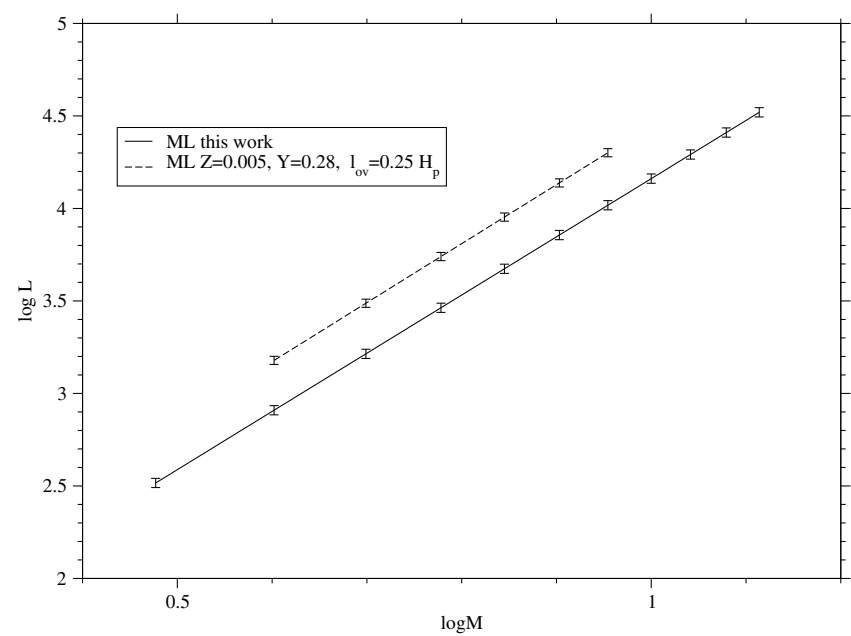

Fig. 8. Comparison between the mass-luminosity relation from Eq. (4) $\left(Y=0.28, Z=0.005, l_{\mathrm{ov}}=0.25 H_{\mathrm{p}}\right)$ and the global $M L$ relation from Eq. (3).

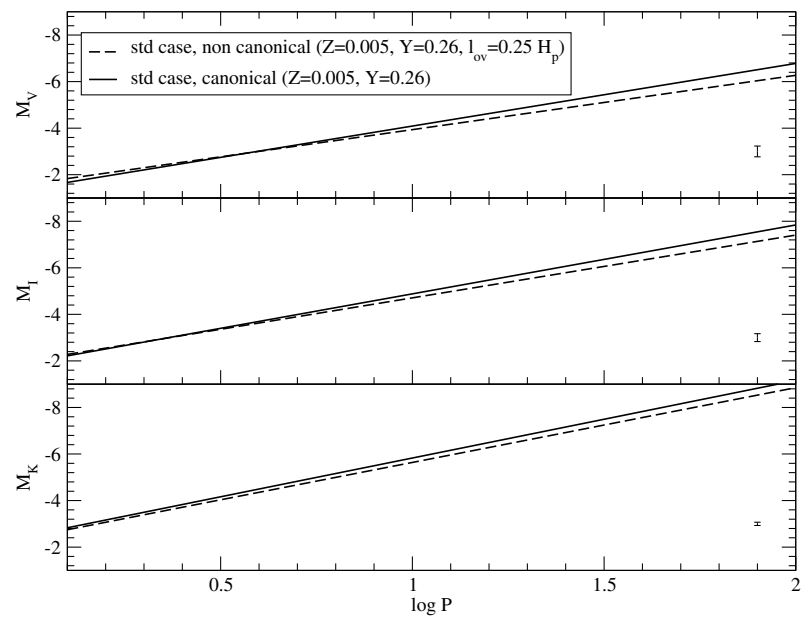

Fig. 9. Comparison, in the $V, I$ and $K$ photometric bands, between the present canonical and noncanonical linear $P L$ relations (solid and dashed lines).

A comparison between the canonical (case std of Table 9) and the noncanonical $P L$ linear relation in the $V, I, K$ bands (see Table 17) is shown in Fig. 9, where the errorbars refer to the intrinsic dispersions of the canonical relations. We notice that both the zero point and the slope of the $P L$ relations are affected by the inclusion of mild overshooting. The noncanonical $V$ band relation predicts brighter magnitudes than the canonical ones in the very short period range and fainter magnitudes than the canonical ones for periods longer than about 5 days, whereas the noncanonical $I$ and $K$ band $P L$ predict almost identical magnitudes to the canonical ones at the shortest periods and fainter magnitudes than their canonical counterparts elsewhere, with the effect increasing toward longer periods. At a period of 10 days, the effect of applying the noncanonical relations instead of the canonical ones would be of about $0.15,0.17$ and $0.19 \mathrm{mag}$ in the $V, I$ and $K$ band respectively, whereas at 30 days it would be as large as about $0.32,0.29$ and 0.24 in the $V, I$ and $K$ band respectively.

Referring to the upper panel of Fig. 7, we report in Table 18 the minimum and maximum masses with blue loops that cross the red edge of the instability strip, together with the corresponding values of the minimum and maximum period.
Table 17. Theoretical $P L$ relations for fundamental noncanonical pulsators (see text).

\begin{tabular}{llll}
\hline \hline Case & $a$ & $b$ & $\sigma$ \\
\hline & & $\overline{M_{V}}$ & \\
$n c O S$ & $-1.60 \pm 0.04$ & $-2.34 \pm 0.03$ & 0.29 \\
$n c O S$ cut & $-1.47 \pm 0.04$ & $-2.47 \pm 0.04$ & 0.28 \\
& & $\overline{M_{I}}$ & \\
$n c O S$ & $-2.01 \pm 0.03$ & $-2.7 \pm 0.02$ & 0.21 \\
$n c O S$ cut & $-1.92 \pm 0.03$ & $-2.8 \pm 0.03$ & 0.20 \\
& & $\overline{M_{K}}$ & \\
$n$ cOS & $-2.43 \pm 0.01$ & $-3.21 \pm 0.01$ & 0.08 \\
$n c O S$ cut & $-2.39 \pm 0.01$ & $-3.25 \pm 0.01$ & 0.08 \\
\hline
\end{tabular}

Table 18. $M_{\min }, M_{\max }, P_{\min }, P_{\max }$ (as in Table 5) for $l_{\mathrm{ov}}=0.25 H_{\mathrm{p}}$.

\begin{tabular}{lllll}
\hline \hline Case & $\begin{array}{l}M_{\min } \\
\left(M_{\odot}\right)\end{array}$ & $\begin{array}{l}M_{\max } \\
\left(M_{\odot}\right)\end{array}$ & $\begin{array}{l}P_{\min } \\
(\text { days })\end{array}$ & $\begin{array}{l}P_{\max } \\
(\text { days })\end{array}$ \\
\hline XII $\left(l_{\mathrm{ov}}=0.25 H_{\mathrm{p}}\right)$ & 4 & 9 & 4.1 & 72.4 \\
\hline
\end{tabular}

\subsection{Mass loss}

The possibility that mass loss might solve the long-standing problem of the mass discrepancy of Cepheids between the value inferred from pulsational and evolutionary models has been suggested by various authors (see e.g. Bono et al. 2002; Caputo et al. 2005). The argument is that in order to solve the problem - that is, to provide higher luminosity for He-burning stars of a given mass - a larger He-core mass is needed. As previously discussed, this is precisely the effect of the convective core-overshooting. On the other hand, a similar result can be mimicked by a standard model affected by significant mass loss. In order to check this, we computed the evolution of a $5 M_{\odot}$ star taking into account the effect of a mild core overshooting $\left(l_{\mathrm{ov}}=0.25 H_{\mathrm{p}}\right)$ during the $\mathrm{H}$-burning phase. We attempted to reproduce the minimum luminosity $\left(L_{\mathrm{He}}^{\mathrm{OV}}\right)$ of this model during the central He-burning by standard models affected by mass loss. The mass loss process is not yet fully understood and it is still lacking satisfactory knowledge of its efficiency along the various evolutionary phases. As a consequence, we are forced to adopt different mass loss prescriptions in the following numerical experiments in order to study the problem. Thus we computed the evolution of stars with mass in the range between 6.0 and $7 M_{\odot}$ with different mass loss prescriptions adopting the classical Schwarzschild criterion to determine the convective core boundary; the results, discussed below, are shown in Fig. 10. The models were evolved with constant mass during the main sequence and subgiant phase, while a substantial mass loss, with a constant rate, was imposed during the red giant phase. Once the total mass of the models reached the desired value, the subsequent evolution was performed at constant mass. Following such a procedure, we computed for example the evolution of the central He-burning phase of a $5.3 M_{\odot}$ with a progenitor star of $6.2 M_{\odot}$, whose behavior in the HR-diagram is like that of our target, the $5 M_{\odot}$ with core overshooting. However, further numerical experiments showed that the result is not unique; models with different initial masses and different prescriptions for the mass loss have a minimum luminosity during the central He-burning very similar to $L_{\mathrm{He}}^{\mathrm{ov}}$. Such a result was expected since the Cepheid $M L$ relation depends appreciably on both the mass-loss rate and the evolutionary phase at which it is turned on. An example is given by a star with initial mass of $6.1 M_{\odot}$ evolved from the ZAMS, with the classical Reimers mass loss rate (with $\eta=1$ ); even if this model 


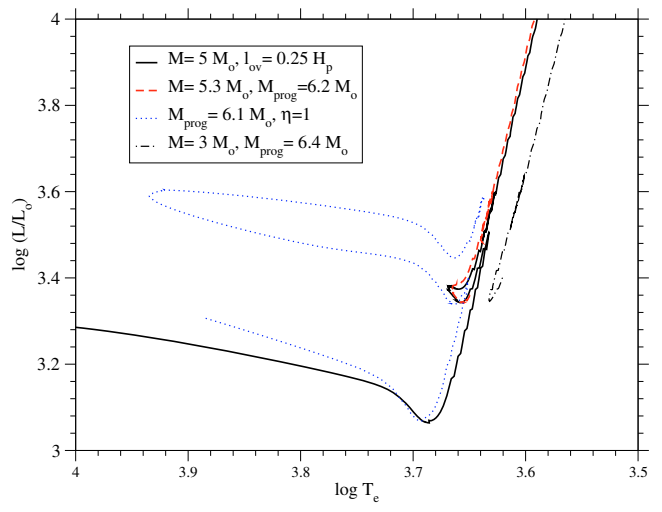

Fig. 10. Comparison of the minimum luminosity during the central He burning phase for a $5 M_{\odot}$ model with $l_{\mathrm{ov}}=0.25 H_{\mathrm{p}}$ and models of different original masses with different massloss prescriptions.

describes a much more extended loop in color in the HRdiagram, the minimum luminosity of the central He burning phase is the same. The target luminosity can also be reproduced by a more extreme model: a $3 M_{\odot}$ with a progenitor of $6.4 M_{\odot}$. In this case, the color of the He-burning model is significantly redder than the model with core overshooting. These few numerical examples prove that it does not make sense to try to compute a $M L$ relationship for standard models with mass loss, unless a priori mass loss prescriptions have been fixed. Moreover, the mass-loss solution of the Cepheid mass discrepancy sounds rather ad hoc, at least until a physical process responsible for the huge required mass loss is found and understood.

\section{Conclusions}

On the basis of an updated set of evolutionary and pulsation models for classical Cepheids in the LMC, we have investigated the effect of the uncertainties on the chemical composition and on the physical assumptions adopted in the codes on the most relevant pulsation observables and thus on the theoretical calibration of the Cepheid distance scale. We found that present uncertainties on relevant nuclear reaction rates have only a negligible effect on evolutionary and pulsational theoretical prediction. Moreover the uncertainties in the metal and helium abundances affect the results of the evolutionary computations but do not significantly change the pulsation scenario. On the other hand, the still present uncertainties on the efficiency of the overshooting phenomenon in the previous $\mathrm{H}$ burning phase and on the mass-loss rates are found to be the most important source of uncertainty in the theoretical Cepheid mass-luminosity and period-luminosity relations. In particular, using a theoretical $P L$ relation that relies on the assumption of mildly overshooting evolutionary models, one infers distances that are significantly shorter than the values obtained when a canonical theoretical $P L$ is used, especially in the optical bands. Therefore we conclude that the uncertainty on the Cepheid $M L$ relation is expected to significantly affect the Cepheid calibration of the extragalactic distance scale and in turn the evaluation of the Hubble constant. The application of the theoretical analysis performed in this paper to LMC Cepheid data will be addressed in a forthcoming paper (Valle et al. 2009, in prep.).

Acknowledgements. We thank our anonymous referee for his/her valuable comments that improved the quality of our paper. We also warmly thank S. Shore for a careful reading of the manuscript. Financial support for this study was provided by PRIN-INAF 2006 (PI G. Clementini).

\section{References}

Alcock, C., \& Paczynski, B. 1978, ApJ, 223, 244

Andrievsky, S. M., Kovtyukh, V. V., Korotin, S. A., Spite, M., \& Spite, F. 2001, A\&A, 367, 605

Angulo, C., Arnould, M., Rayet, M., et al. 1999, Nucl. Phys. A, 656, 3

Asplund, M., Grevesse, N., \& Sauval, A. J. 2005, in Cosmic Abundances as Records of Stellar Evolution and Nucleosynthesis, ed. F. N. Bash, \& T. J. Barnes, ASP Conf. Ser., 336, 25

Badnell, N. R., Bautista, M. A., Butler, K., et al. 2005, MNRAS, 360, 458

Bahcall, J. N., Basu, S., Pinsonneault, M., \& Serenelli, A. M. 2005, ApJ, 618, 1049

Barmina, R., Girardi, L., \& Chiosi, C. 2002, A\&A, 385, 847

Basu, S., \& Antia, H. M. 2004, ApJ, 606L, 85

Beaulieu, J. P., Buchler, J. R., \& Kollath, Z. 2001, A\&A, 373, 164

Bemmerer, D., Confortola, F., Lemut, A., et al. 2006, Nucl. Phys. A, 779, 297

Bessel, M. S., Castelli, F., \& Plez, B. 1998, A\&A, 333, 231

Böhm-Vitense, E. 1958, Zs.f.Ap., 46, 108

Bono, G., \& Stellingwerf, R. F. 1994, ApJS, 93, 233

Bono, G., Marconi, M., \& Stellingwerf, R. F. 1997, ApJ, 477, 346

Bono, G., Marconi, M., \& Stellingwerf, R. F. 1999a, ApJS, 122, 167

Bono, G., Caputo, F., Castellani, V., \& Marconi, M. 1999b, ApJ, 512, 711

Bono, G., Caputo, F., Cassisi, S., et al. 2000, ApJ, 543, 955

Bono, G., Gieren, W. P., Marconi, M., Fouqué, P., \& Caputo, F. 2001, ApJ, 563, 319

Bono, G., Castellani, V., \& Marconi, M. 2002, ApJ, 565, L83

Bono, G., Caputo, F., Fiorentino, G., Marconi, M., \& Musella, I. 2008, ApJ, 684, 102

Brocato, E., \& Castellani, V. 1993, ApJ, 410, 99

Brocato, E., Castellani, V., Di Carlo, E., Raimondo, G., \& Walker, A. R. 2003, AJ, 125, 3111

Brocato, E., Caputo, F., Castellani, V., Marconi, M., \& Musella, I. 2004, AJ, 128, 1597

Brunish, W. M., \& Becker, S. A. 1990, ApJ, 351, 258

Buchmann, L. R., \& Barnes, C. A. 2006, Nucl. Phys. A, 777, 254

Caffau, E., Ludwig, H. G., Steffen, M., et al. 2008, A\&A, 488, 1031

Caldwell, J. A. R., \& Coulson, I. M. 1986, MNRAS, 218, 223

Caldwell, J. A. R., \& Laney, C. D. 1991, in The Magellanic Clouds, IAU Symp., 148,249

Canuto, V. M. 2000, ApJ, 534, L113

Caputo, F., Chieffi, A., Tornambé, A., Castellani, V., \& Pulone, L. 1989, ApJ, 340,241

Caputo, F., Marconi, M., \& Musella, I. 2000, A\&A, 354, 610

Caputo, F., Bono, G., Fiorentino, G., Marconi, M., \& Musella, I. 2005, ApJ, 629, 1021

Castellani, V., Giannone, P., \& Renzini, A. 1971a, Ap\&SS, 10, 340

Castellani, V., Giannone, P., \& Renzini, A. 1971b, Ap\&SS, 10, 355

Castellani, V., Chieffi, A., Tornambé, A., \& Pulone, L. 1985, ApJ, 296, 204

Castellani, V., Chieffi, A., \& Straniero, O. 1990, ApJS, 74, 463

Castellani, V., Chieffi, A., \& Straniero, O. 1992, ApJS, 78, 517

Chieffi, A., \& Straniero, O. 1989, ApJS, 71, 47

Chin, C., \& Stothers, R. B. 1991, ApJS, 77, 299

Chiosi, C., \& Maeder, A. 1986, ARA\&A, 24, 329

Chiosi, C., Bertelli, S., \& Bressan, A. 1992, ARA\&A, 30, 305

Chiosi, C., Wood, P. R., \& Capitanio, N. 1993, ApJS, 86, 541

Ciacio, F., Degl'Innocenti, S., \& Ricci, B. 1997, A\&AS, 123, 449

Claret, A. 2007, A\&A, 475, 1019

Cordier, D., Lebreton, Y., Goupil, M. J., et al. 2002, A\&A, 392, 169

Cox, J. P., \& Giuli, R. T. 1968, Principles of stellar Structure (New York, USA: Gordon and Breach Science publishers)

Degl'Innocenti, S., Prada Moroni, P. G., \& Ricci, B. 2006, Ap\&SS, 305, 67

Degl'Innocenti, S., Prada Moroni, P. G., Marconi, M., \& Ruoppo, A. 2008, Ap\&SS, 316, 25

Demarque, P., Robinson, F. J., \& Straka, C. W. 2007, in From Stars to Galaxies: Building the Pieces to Build Up the Universe, ed. A. Vallenari, R. Tantalo, L. Portinari, \& A. Moretti, ASP Conf. Ser., 374, 27

Di Criscienzo, M., Marconi, M., \& Caputo, F. 2004, ApJ, 612, 1092

Dorman, B., \& Rood, R. T. 1993, ApJ, 409, 387

Eggleton, P. P., Dearborn, D. S., \& Lattanzio, J. C. 2007, ApJ, 677, 581

Feast, M. W. 1984, in Structure and evolution of the Magellanic Clouds, IAU Symp., 108, 157

Feast, M. W. 1991, in The Magellanic Clouds, IAU Symp., 148, 1

Feast, M. W. 1995, PASP, 111, 775

Ferguson, J. W., Alexander, D. R., Allard, F., et al. 2005, ApJ, 623, 585

Fiorentino, G., Caputo, F., Marconi, M., \& Musella, I. 2002, ApJ, 576, 402

Fiorentino, G., Marconi, M., Musella, I., \& Caputo, F. 2007, A\&A, 476, 863

Freedman, W. L., Madore, B. F., Gibson, B. K., et al. 2001, ApJ, 553, 47

Fynbo, H. O. U., Diget, C. A., Bergmann, U. C., et al. 2005, Nature, 433, 136 
Gennaro, M., Prada Moroni, P. G., \& Degl'Innocenti, S. 2008, proceedings of $52^{\circ}$ congresso SAIt, Mem. Soc. Astron. Ital., in publication

Girardi, L., Bressan, A., Bertelli, G., \& Chiosi, C. 2000, A\&AS, 141, 371

Graboske, H. C., Dewitt, H. E., Grossman, A. S., \& Cooper, M. 1973, ApJ, 181, 457

Grevesse, N., \& Noels, A. 1993, in Origin and Evolution of the elements, ed. N. Prantzos, E. Vangioni-Flam, M. Cassé (Cambridge: Cambridge Univ. Press), 15

Grevesse, N., \& Sauval, A. J. 1998, Space Sci. Rev., 85, 161

Hammer, J. W., Fey, M., Kunz, R., et al. 2005, Nucl. Phys. A, 758, 363

Huang, R. Q., \& Weigert, A. 1983, ApJ, 127, 309

Iben, I. 1972, ApJ, 178, 433

Iglesias, C., \& Rogers, F. J. 1996, ApJ, 464, 943

Imbriani, G., Costantini, H., Formicola, A., et al. 2005, Eur. Phys. J. A, 25, 455

Itoh, N., Totsuji, H., \& Ichimaru, S. 1977, ApJ, 218, 477

Itoh, N., Totsuji, H., Ichimaru, S., \& Dewitt, H. E. 1979, ApJ, 234, 1079

Izotov, Y. I., Thuan, T. X., \& Stasińska, G. 2007, ApJ, 662, 15

Jimenez, R., Flynn, C., MacDonald, J., \& Gibson, B. K. 2003, Science, 299, 1552

Kanbur, S. M., \& Ngeow, C.-C. 2006, MNRAS, 369, 705

Kanbur, S. M., Ngeow, C.-C., \& Buchler, J. R. 2004, MNRAS, 354, 212

Keller, S. 2008, ApJ, 677, 483

Kennicutt, R. C., Jr., Stetson, P. B., Saha, A., et al. 1998, ApJ, 498, 181

Koen, C., Kanbur, S., \& Ngeow, C. 2007, MNRAS, 380, 1440

Korn, A. J., Becker, S. R., Gummersbach, C. A., \& Wolf, B. 2000, A\&A, 353, 655

Lauterborn, D., Refsdal, S., \& Weigert, A. 1971, A\&A, 10, 97

Laney, C. D., \& Stobie, R. S. 1986, MNRAS, 222, 449

Lemut, A., Bemmerer, D., Confortola, F., et al. 2006, Phys. Lett. B, 634, 483

Luck, R. E., Moffett, T. J., Barnes, T. G. III, \& Gieren, W. P. 1998, AJ, 115, 605

Macri, L. M., Stanek, K. Z., Bersier, D., Greenhill, L. J., \& Reid, M. J. 2006, ApJ, 652, 1133

Madore, B., \& Freedman, W. L. 1991, PASP, 103, 933

Maeder, A., \& Zahn, J. P. 1998, A\&A, 334, 1000

Marconi, M., \& Di Criscienzo, M. 2007, MNRAS, 467, 223

Marconi, M., Caputo, F., Di Criscienzo, M., \& Castellani, M. 2003, ApJ, 596, 299

Marconi, M., Fiorentino, G., \& Caputo, F. 2004, A\&A, 417, 1101

Marconi, M., Musella, I., \& Fiorentino, G. 2005, ApJ, 632, 590

Matraka, B., Wassermann, C., \& Weigert, A. 1982, A\&A, 107, 283

Maeder, A., \& Meynet, G. 2000, A\&A, 361, 159

Meakin, C. A., \& Arnett, D. 2007, ApJ, 667, 448

Meynet, G., \& Maeder, A. 2000, A\&A, 361, 101

Natale, G., Marconi, M., \& Bono, G. 2008, ApJL, 674, 93

Ngeow, C.-C., Kanbur, S. M., Nikolaev, S., et al. 2005, MNRAS, 363, 831
Neuforge-Verheecke, C., Guzik, J. A., Keady, J. J., et al. 2001, ApJ, 561, 450 Noels, A., \& Gabriel, M. 1973, A\&A, 24, 201

Pagel, B. E. J., \& Portinari, L. 1998, MNRAS, 298, 747

Palacios, A., Talon, S., Charbonnel, C., \& Forestini, M. 2003, A\&A, 399, 603

Peimbert, M., Luridiana, V., Peimbert, A., \& Carigi, L. 2007, ASP Conf. Ser., 374, 81

Persson, S. E., Madore, B. F., Krzemiński, W., et al. 2004, AJ, 128, 2239

Potekhin, A. Y. 1999, A\&A, 351, 787

Petroni, S., Bono, G., Marconi, M., \& Stellingwerf, R. F. 2003, ApJ, 599, 522

Renzini, A., \& Ritossa, C. 1994, ApJ, 433, 293

Renzini, A., Greggio, L., \& Ritossa, C. 1992, ApJ, 400, 280

Riello, M., Cassisi, S., Piotto, G., et al. 2003, A\&A, 410, 553

Robertson, J. W. 1971, ApJ, 170, 353

Rogers, F. J., Swenson, F. J., \& Iglesias, C. A. 1996, ApJ, 456, 902

Romaniello, M., Primas, F., Mottini, M., et al. 2005, A\&A, 429, 37

Romaniello, M., Primas, F., Mottini, M., et al. 2008, A\&A, 488, 731

Saha, A., Sandage, A., Tammann, G. A., et al. 2001, ApJ, 562, 314

Sakai, S., Ferrarese, L., Kennicutt, R. C. Jr., \& Saha, A. 2004, ApJ, 608, 42

Salasnich, B., Bressan, A., \& Chiosi, C. 1999, A\&A, 342, 131

Salpeter, E. E. 1954, Aust. J. Phys., 7, 373

Sandage, A., Tammann, G. A., \& Reindl, B. 2004, A\&A, 424, 43

Sasselov, D. D., Beaulieu, J. P., Renault, C., et al. 1997, A\&A, 324, 471

Sestito, P., Castellani, V., Degl'Innocenti, S., \& Prada Moroni, P. G. 2002, in Observed HR diagrams and stellar evolution: the interplay between observational constraints and theory, ed. T. Lejeune, \& J. Fernandes, ASP Conf. Ser., 274, 73

Shternin, P. S., \& Yakovlev, D. G. 2006, PhRvD, 74(4), 3004

Stothers, R. B. 1988, ApJ, 329, 712

Stothers, R. B., \& Chin, C. 1991a, ApJ, 374, 288

Stothers, R. B., \& Chin, C. 1991b, ApJ, 381, L67

Stothers, R. B., \& Chin, C. 1992, ApJ, 390, L33

Stothers, R. B., \& Chin, C. 1993, ApJ, 412, 294

Stothers, R. B., \& Chin, C. 1994, ApJ, 421, L91

Straka, C. W., Demarque, P., \& Guenther, D. B. 2005, ApJ, 629, 107

Sweigart, A., \& Demarque, P. 1972, A\&A, 20, 445

Sweigart, A., \& Demarque, P. 1973, in Variable Stars in Globular Clusters and in Related Systems, ed. J. D. Fernie, \& D. Reidel, IAU Coll., 21, 221

Udalski, A., Szymanski, M., Kubiak, M., et al. 1999, AcA, 49, 201

Xu, H. Y., \& Li, Y. 2004a, A\&A, 418, 213

Xu, H. Y., \& Li, Y. 2004b, A\&A, 418, 225

Vázquez, G. A., et al. 2005, ApJ, 663, 995

Vemury, S. K., \& Stothers, R. 1978, ApJ, 225, 939

Weiss, A., Serenelli, A., Kitsikis, A., Schlattl, H., \& Christensen-Dalsgaard, J. 2005, A\&A, 441, 1129 
G. Valle et al.: Uncertainties on classical Cepheid predictions, Online Material $p 1$

Table 2. Coefficients of the linear analytical relations for the fundamental red and blue edges.

\begin{tabular}{llll}
\hline \hline Case & $a$ & $b$ & $\sigma$ \\
\hline Blue edge & & & \\
I $($ std $)$ & $3.848 \pm 0.010$ & $-0.022 \pm 0.002$ & 0.005 \\
II $\left(Z_{\text {low }}\right)$ & $3.828 \pm 0.013$ & $-0.016 \pm 0.003$ & 0.006 \\
III $\left(Z_{\text {high }}\right)$ & $3.853 \pm 0.029$ & $-0.025 \pm 0.008$ & 0.012 \\
IV $\left(Y_{\text {high }}\right)$ & $3.866 \pm 0.020$ & $-0.026 \pm 0.005$ & 0.009 \\
V $\left(Y_{\text {high }}, Z_{\text {high }}\right)$ & $3.870 \pm 0.020$ & $-0.029 \pm 0.006$ & 0.008 \\
VI $\left(Z_{\text {high }}, \mathrm{GS} 98\right)$ & $3.858 \pm 0.022$ & $-0.027 \pm 0.007$ & 0.011 \\
Red edge & & & \\
I $($ std $)$ & $3.955 \pm 0.020$ & $-0.073 \pm 0.005$ & 0.010 \\
II $\left(Z_{\text {low }}\right)$ & $3.922 \pm 0.048$ & $-0.061 \pm 0.012$ & 0.023 \\
III $\left(Z_{\text {high }}\right)$ & $3.974 \pm 0.028$ & $-0.082 \pm 0.008$ & 0.012 \\
IV $\left(Y_{\text {high }}\right)$ & $3.934 \pm 0.021$ & $-0.066 \pm 0.005$ & 0.009 \\
V $\left(Y_{\text {high }}, Z_{\text {high }}\right)$ & $3.965 \pm 0.019$ & $-0.078 \pm 0.005$ & 0.008 \\
VI $\left(Z_{\text {high }}, \mathrm{GS} 98\right)$ & $3.958 \pm 0.018$ & $-0.074 \pm 0.005$ & 0.009 \\
\hline
\end{tabular}

Table 6. $M L$ relations for the selected cases; $\log \left(L / L_{\odot}\right)=a+$ $b \log \left(M / M_{\odot}\right)$.

\begin{tabular}{llll}
\hline \hline Case & $a$ & $b$ & $\sigma$ \\
\hline I $($ std $)$ & $1.000 \pm 0.017$ & $3.160 \pm 0.019$ & 0.009 \\
II $\left(Z_{\text {low }}\right)$ & $1.040 \pm 0.010$ & $3.110 \pm 0.011$ & 0.008 \\
III $\left(Z_{\text {high }}\right)$ & $0.736 \pm 0.008$ & $3.455 \pm 0.010$ & 0.002 \\
IV $\left(Y_{\text {high }}\right)$ & $1.095 \pm 0.043$ & $3.074 \pm 0.047$ & 0.024 \\
\hline
\end{tabular}


G. Valle et al.: Uncertainties on classical Cepheid predictions, Online Material $p 2$

Table 7. Canonical $P L T_{\mathrm{e}}$ relations for the selected cases; $\log P=a+b \log \left(L / L_{\odot}\right)+c\left(\log T_{\mathrm{e}}\right)$.

\begin{tabular}{lllll}
\hline \hline Case & $a$ & $b$ & $c$ & $\sigma$ \\
\hline Fundamental - CANONICAL & & & & \\
I $(s t d)$ & $10.725 \pm 0.180$ & $0.687 \pm 0.004$ & $-3.261 \pm 0.046$ & 0.013 \\
II $\left(Z_{\text {low }}\right)$ & $10.475 \pm 0.320$ & $0.696 \pm 0.005$ & $-3.202 \pm 0.082$ & 0.016 \\
III $\left(Z_{\text {high }}\right)$ & $10.908 \pm 0.173$ & $0.677 \pm 0.004$ & $-3.303 \pm 0.044$ & 0.009 \\
IV $\left(Y_{\text {high }}\right)$ & $11.265 \pm 0.270$ & $0.681 \pm 0.005$ & $-3.398 \pm 0.069$ & 0.013 \\
First Overtone - CANONICAL & & & & \\
I $(s t d)$ & $10.827 \pm 0.393$ & $0.633 \pm 0.004$ & $-3.282 \pm 0.101$ & 0.003 \\
II $\left(Z_{\text {low }}\right)$ & $10.826 \pm 0.187$ & $0.631 \pm 0.002$ & $-3.279 \pm 0.048$ & 0.002 \\
III $\left(Z_{\text {high }}\right)$ & $11.033 \pm 0.404$ & $0.630 \pm 0.003$ & $-3.337 \pm 0.105$ & 0.004 \\
IV $\left(Y_{\text {high }}\right)$ & $10.897 \pm 0.347$ & $0.633 \pm 0.003$ & $-3.299 \pm 0.090$ & 0.002 \\
\hline
\end{tabular}


G. Valle et al.: Uncertainties on classical Cepheid predictions, Online Material p 3

Table 10. Theoretical linear period-luminosity relations in different photometric bands for individual cases.

\begin{tabular}{|c|c|c|c|}
\hline Case & 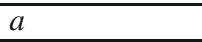 & $\bar{b}$ & $\bar{\sigma}$ \\
\hline & & $\overline{M_{B}}$ & \\
\hline$Z_{\text {low }}$ & $-0.72 \pm 0.02$ & $-2.61 \pm 0.02$ & 0.27 \\
\hline$Z_{\text {high }}$ & $-1.00 \pm 0.03$ & $-2.18 \pm 0.04$ & 0.29 \\
\hline$Y_{\text {high }}$ & $-1.03 \pm 0.03$ & $-2.28 \pm 0.03$ & 0.37 \\
\hline & & $\overline{M_{V}}$ & \\
\hline$Z_{\text {low }}$ & $-1.16 \pm 0.01$ & $-2.90 \pm 0.01$ & 0.20 \\
\hline$Z_{\text {high }}$ & $-1.40 \pm 0.02$ & $-2.61 \pm 0.03$ & 0.21 \\
\hline$Y_{\text {high }}$ & $-1.39 \pm 0.02$ & $\frac{-2.68}{M_{R}} \pm 0.02$ & 0.27 \\
\hline$Z_{\text {low }}$ & $-1.44 \pm 0.01$ & $-3.00 \pm 0.01$ & 0.16 \\
\hline$Z_{\text {high }}$ & $-1.66 \pm 0.02$ & $-2.77 \pm 0.02$ & 0.18 \\
\hline$Y_{\text {high }}$ & $-1.63 \pm 0.02$ & $\frac{-2.82}{M_{I}} \pm 0.02$ & 0.23 \\
\hline$Z_{\text {low }}$ & $-1.75 \pm 0.01$ & $-3.10 \pm 0.01$ & 0.14 \\
\hline$Z_{\text {high }}$ & $-1.93 \pm 0.02$ & $-2.90 \pm 0.02$ & 0.15 \\
\hline$Y_{\text {high }}$ & $-1.90 \pm 0.02$ & $\frac{-2.95}{M_{J}} \pm 0.02$ & 0.20 \\
\hline$Z_{\text {low }}$ & $-2.117 \pm 0.006$ & $-3.240 \pm 0.007$ & 0.10 \\
\hline$Z_{\text {high }}$ & $-2.247 \pm 0.011$ & $-3.119 \pm 0.014$ & 0.11 \\
\hline$Y_{\text {high }}$ & $-2.217 \pm 0.013$ & $\frac{-3.140 \pm 0.013}{M_{K}}$ & 0.14 \\
\hline$Z_{\text {low }}$ & $-2.430 \pm 0.003$ & $-3.373 \pm 0.004$ & 0.06 \\
\hline$Z_{\text {high }}$ & $-2.507 \pm 0.007$ & $-3.317 \pm 0.009$ & 0.07 \\
\hline$Y_{\text {high }}$ & $-2.477 \pm 0.008$ & $-3.320 \pm 0.008$ & 0.09 \\
\hline
\end{tabular}

Table 12. Theoretical quadratic period-luminosity relations for fundamental pulsators in the various individual cases.

\begin{tabular}{lllll}
\hline \hline Case & $a$ & $b$ & $c$ & $\sigma$ \\
\hline$Z_{\text {low }}$ & $-0.62 \pm 0.03$ & $-2.92 \pm 0.08$ & $0.18 \pm 0.04$ & 0.27 \\
$Z_{\text {high }}$ & $-0.25 \pm 0.08$ & $-4.32 \pm 0.22$ & $1.35 \pm 0.14$ & 0.27 \\
$Y_{\text {high }}$ & $-0.74 \pm 0.10$ & $-2.92 \pm 0.20$ & $0.30 \pm 0.09$ & 0.36 \\
& & $\overline{M_{V}}$ & & \\
$Z_{\text {low }}$ & $-1.10 \pm 0.02$ & $-3.08 \pm 0.06$ & $0.11 \pm 0.03$ & 0.20 \\
$Z_{\text {high }}$ & $-0.87 \pm 0.06$ & $-4.12 \pm 0.16$ & $0.96 \pm 0.10$ & 0.20 \\
$Y_{\text {high }}$ & $-1.19 \pm 0.07$ & $-3.09 \pm 0.14$ & $0.20 \pm 0.07$ & 0.27 \\
& & $\overline{M_{R}}$ & & \\
$Z_{\text {low }}$ & $-1.40 \pm 0.02$ & $-3.15 \pm 0.05$ & $0.08 \pm 0.03$ & 0.16 \\
$Z_{\text {high }}$ & $-1.21 \pm 0.05$ & $-4.05 \pm 0.14$ & $0.81 \pm 0.09$ & 0.17 \\
$Y_{\text {high }}$ & $-1.47 \pm 0.06$ & $-3.17 \pm 0.12$ & $0.16 \pm 0.06$ & 0.23 \\
& & $\bar{M} I$ & & \\
$Z_{\text {low }}$ & $-1.71 \pm 0.02$ & $-3.22 \pm 0.04$ & $0.07 \pm 0.02$ & 0.14 \\
$Z_{\text {high }}$ & $-1.54 \pm 0.04$ & $-4.01 \pm 0.12$ & $0.70 \pm 0.07$ & 0.14 \\
$Y_{\text {high }}$ & $-1.76 \pm 0.05$ & $-3.25 \pm 0.10$ & $0.14 \pm 0.05$ & 0.19 \\
& & $\bar{M} J$ & & \\
$Z_{\text {low }}$ & $-2.09 \pm 0.01$ & $-3.32 \pm 0.03$ & $0.04 \pm 0.02$ & 0.10 \\
$Z_{\text {high }}$ & $-1.97 \pm 0.03$ & $-3.90 \pm 0.08$ & $0.50 \pm 0.05$ & 0.10 \\
$Y_{\text {high }}$ & $-2.13 \pm 0.04$ & $-3.34 \pm 0.08$ & $0.09 \pm 0.04$ & 0.14 \\
& & $\overline{M_{K}}$ & & \\
$Z_{\text {low }}$ & $-2.42 \pm 0.01$ & $-3.41 \pm 0.02$ & $0.02 \pm 0.01$ & 0.06 \\
$Z_{\text {high }}$ & $-2.34 \pm 0.02$ & $-3.80 \pm 0.05$ & $0.31 \pm 0.03$ & 0.07 \\
$Y_{\text {high }}$ & $-2.43 \pm 0.02$ & $-3.43 \pm 0.05$ & $0.05 \pm 0.02$ & 0.09 \\
\hline & & & &
\end{tabular}


G. Valle et al.: Uncertainties on classical Cepheid predictions, Online Material p 4

Table 14. Non canonical $P L T_{\mathrm{e}}$ relations: $\log P=a+b \log \left(L / L_{\odot}\right)+c\left(\log T_{\mathrm{e}}\right)$.

\begin{tabular}{|c|c|c|c|c|}
\hline Case & $\bar{a}$ & $\bar{b}$ & $\bar{c}$ & $\overline{\sigma \sigma}$ \\
\hline $\begin{array}{l}\text { Fundamental - Non canonical } \\
\text { I }(\text { std) }\end{array}$ & $10.290 \pm 0.290$ & $0.701 \pm 0.005$ & $-3.144 \pm 0.075$ & 0.018 \\
\hline $\begin{array}{l}\text { First Overtone - Non canonical } \\
\text { I }(s t d)\end{array}$ & $10.840 \pm 0.130$ & $0.639 \pm 0.002$ & $-3.278 \pm 0.032$ & 0.001 \\
\hline
\end{tabular}


G. Valle et al.: Uncertainties on classical Cepheid predictions, Online Material p 5

Table 15. Theoretical linear period-luminosity relations for the fundamental noncanonical pulsators.

\begin{tabular}{llll}
\hline \hline Case & $a$ & $b$ & $\sigma$ \\
\hline & & $\overline{M_{B}}$ & \\
$n c O S$ & $-1.40 \pm 0.05$ & $-1.81 \pm 0.04$ & 0.41 \\
$n c O S$ cut & $-1.22 \pm 0.06$ & $-2.00 \pm 0.06$ & 0.40 \\
& & $\overline{M_{R}}$ & \\
$n c O S$ & $-1.79 \pm 0.03$ & $-2.54 \pm 0.03$ & 0.25 \\
$n c O S$ cut & $-1.68 \pm 0.04$ & $-2.65 \pm 0.03$ & 0.24 \\
& & $\overline{M_{J}}$ & \\
$n c O S$ & $-2.24 \pm 0.02$ & $-2.96 \pm 0.02$ & 0.14 \\
$n c O S$ cut & $-2.18 \pm 0.02$ & $-3.03 \pm 0.02$ & 0.14 \\
\hline
\end{tabular}

Table 16. Theoretical quadratic period-luminosity relations for fundamental noncanonical pulsators.

\begin{tabular}{lllll}
\hline \hline Case & $a$ & $b$ & $c$ & $\sigma$ \\
\hline & & $\overline{M_{B}}$ & & \\
$n c O S$ & $-0.87 \pm 0.20$ & $-2.78 \pm 0.36$ & $0.41 \pm 0.15$ & 0.41 \\
$n c O S$ cut & $-2.45 \pm 0.29$ & $0.42 \pm 0.56$ & $-1.13 \pm 0.26$ & 0.39 \\
& & $\overline{M_{V}}$ & & \\
$n c O S$ & $-1.23 \pm 0.15$ & $-3.01 \pm 0.26$ & $0.29 \pm 0.11$ & 0.29 \\
$n c O S$ cut & $-2.39 \pm 0.21$ & $-0.67 \pm 0.4$ & $-0.84 \pm 0.19$ & 0.28 \\
& & $\overline{M_{R}}$ & & \\
$n c O S$ & $-1.47 \pm 0.12$ & $-3.12 \pm 0.22$ & $0.25 \pm 0.09$ & 0.25 \\
$n c O S$ cut & $-2.44 \pm 0.17$ & $-1.14 \pm 0.33$ & $-0.7 \pm 0.16$ & 0.24 \\
& & $\overline{M_{I}}$ & & \\
$n c O S$ & $-1.72 \pm 0.10$ & $-3.22 \pm 0.18$ & $0.22 \pm 0.08$ & 0.21 \\
$n c O S$ cut & $-2.54 \pm 0.15$ & $-1.56 \pm 0.28$ & $-0.58 \pm 0.13$ & 0.2 \\
& & $\overline{M_{J}}$ & & \\
$n c O S$ & $-2.07 \pm 0.07$ & $-3.28 \pm 0.13$ & $0.14 \pm 0.05$ & 0.14 \\
$n c O S$ cut & $-2.64 \pm 0.10$ & $-2.13 \pm 0.20$ & $-0.42 \pm 0.09$ & 0.14 \\
& & $\overline{M_{K}}$ & & \\
$n c O S$ & $-2.35 \pm 0.04$ & $-3.36 \pm 0.07$ & $0.06 \pm 0.03$ & 0.08 \\
$n c O S$ cut & $-2.66 \pm 0.06$ & $-2.72 \pm 0.11$ & $-0.25 \pm 0.05$ & 0.08 \\
\hline
\end{tabular}

